volume 9

ISSUE 1

2021 febbraio

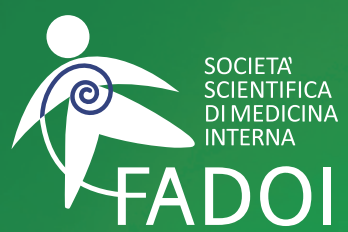

FEDERAZIONE

DELLE ASSOCIAZIONI

DEI DIRIGENTI

OSPEDALIERI

INTERNISTI

Editor in Chief

Michele Meschi

Supervisor Editor

Roberto Nardi

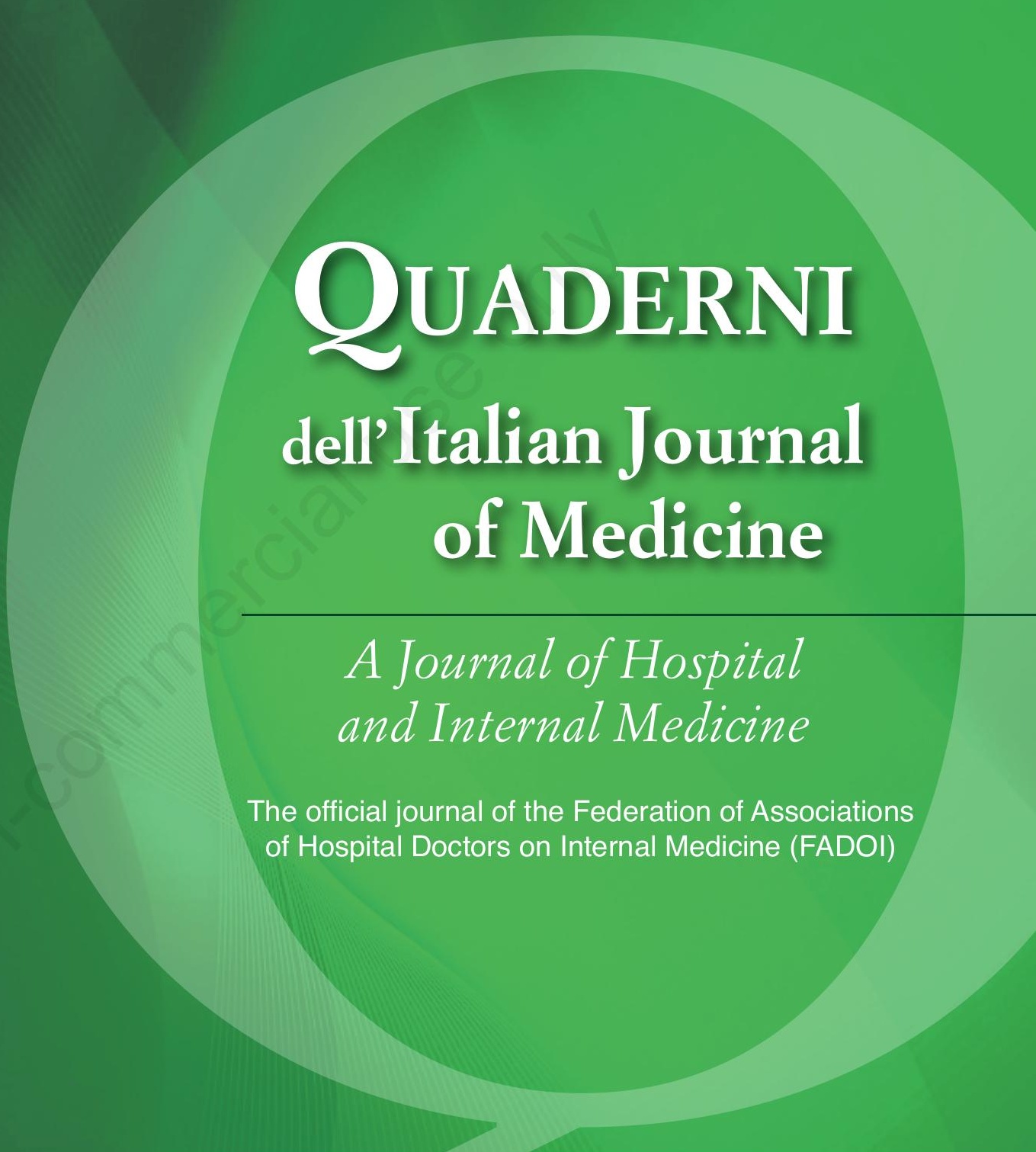

Stipsi indotta da oppioidi: un problema emergente con nuove opportunità terapeutiche

Guest Editors: Franco Radaelli, Dhanai Di Paolo 


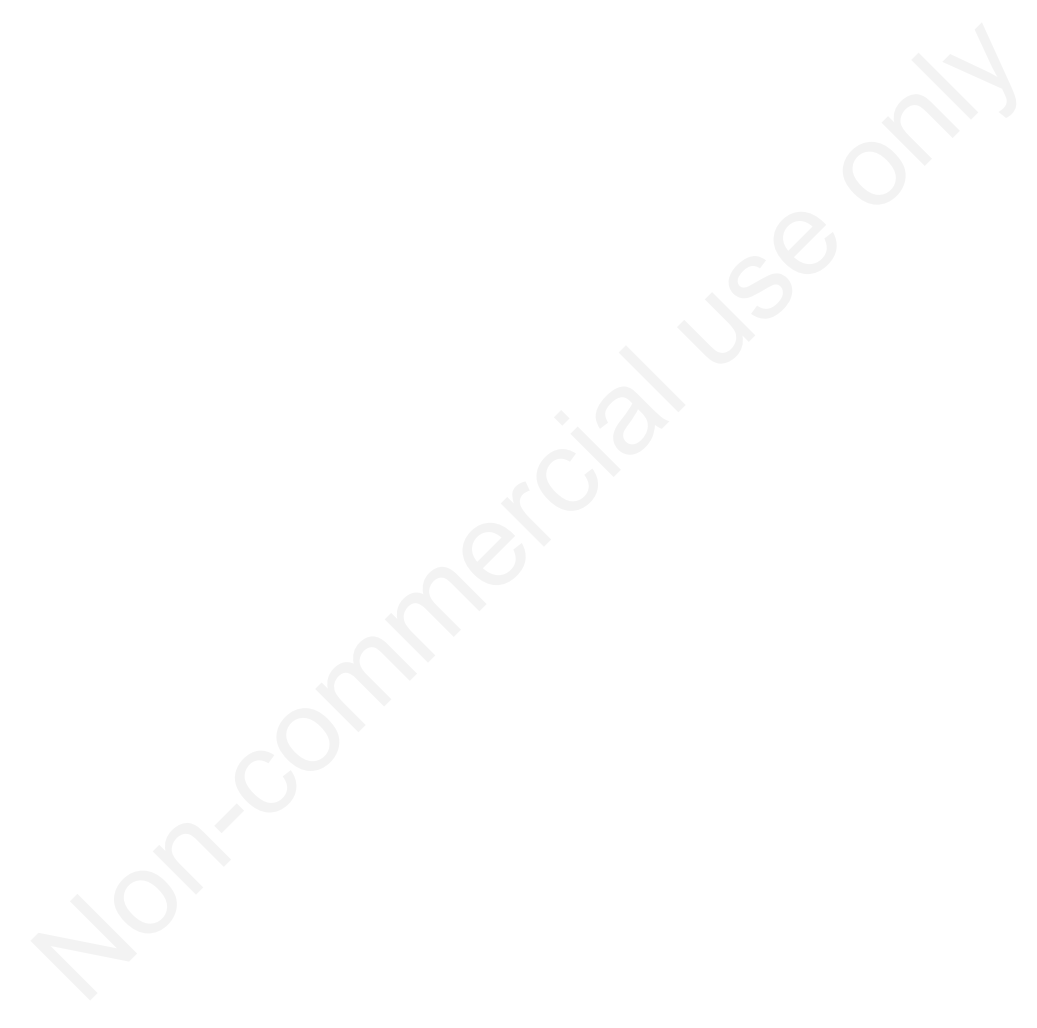




\section{PRESIDENTE ELETTO}

Francesco Dentali, Varese, Italy

\section{PAST PRESIDENT}

Andrea Fontanella, Napoli, Italy

\section{SEGRETARIO}

Paola Gnerre, Savona, Italy

\section{STAFF DI SEGRETERIA}

Lorenza Lenzi, Pomarolo (TN), Italy

Ada Maffettone, Napoli, Italy

Claudia Tieri, Bari, Italy

\section{TESORIERE}

Giorgio Ballardini, Rimini, Italy

COORDINATORE COMMISSIONE GIOVANI

Ombretta Para, Firenze, Italy

\section{RESPONSABILE RAPPORTI ISTITUZIONALI}

Claudio Santini, Roma, Italy

RESPONSABILE RAPPORTI CON LE REGIONI

Alberto Fortini, Firenze, Italy

RESPONSABILE EVENTI E INIZIATIVE SPECIALI

Mauro Campanini, Novara, Italy

EDITOR IN CHIEF ITALIAN JOURNAL OF MEDICINE

Giorgio Vescovo, Padova, Italy
RESPONSABILE DEI

QUADERNI DELL'ITALIAN JOURNAL OF MEDICINE EDIZIONI ON LINE

Michele Meschi, Borgo Val di Taro (PR), Italy

SUPERVISOR EDITOR DEI

QUADERNI DELL'ITALIAN JOURNAL OF MEDICINE

Roberto Nardi, Bologna, Italy

\section{RESPONSABILE SITO WEB E COMUNICAZIONE SOCIAL}

Salvatore Lenti, Arezzo, Italy

Giuseppe Oteri, Milano, Italy

\section{WEB MANAGER E CONTENT EDITOR}

Giuseppe Oteri, Milano, Italy

Davide Ghilardi, Milano, Italy

\section{RESPONSABILE SISTEMA GESTIONE QUALITÀ}

Franco Berti, Roma, Italy

DPO

Alba Sciascera, Magenta (MI), Italy

\section{CONSULTA DEI PRESIDENTI}

Sandro Fontana, Biella, Italy

Salvatore Di Rosa, Palermo, Italy

Ido Iori, Reggio Emilia, Italy

Antonino Mazzone, Legnano (MI), Italy

Carlo Nozzoli, Firenze, Italy

Mauro Campanini, Novara, Italy

Andrea Fontanella, Napoli, Italy 


\section{Italian Journal of Medicine}

A Journal of Hospital and Internal Medicine

\section{COORDINATORE}

David Terracina, Roma, Italy

\section{SEGRETARIO E RESPONSABILE SCIENTIFICO} DEL PROVIDER ECM

Andrea Montagnani, Grosseto, Italy

DIRETTORI DEL DIPARTIMENTO

DELLA RICERCA CLINICA FADOI

Filippo Pieralli, Firenze, Italy

Fulvio Pomero, Savigliano $(C N)$, Italy

DIRETTORI DIPARTIMENTO

PER LA FORMAZIONE E AGGIORNAMENTO

Luigi Magnani, Voghera (PV), Italy

Roberta Re, Novara, Italy

\section{COORDINATORE SCIENTIFICO CENTRO STUDI} FONDAZIONE FADOI

Gualberto Gussoni, Milano, Italy

\section{DELEGATI SOCIETÀ SCIENTIFICHE COLLEGATE}

FISM Antonino Mazzone, Legnano (MI), Italy

SIF Luigi Magnani, Voghera (PV), Italy

Consulta Michele Stornello, Siracusa, Italy

cardiovascolare

SIIA e ISO Michele Stornello, Siracusa, Italy

Arcangelo Iannuzzi, Pomigliano d'Arco (NA), Italy

EFIM

Antonio Brucato, Milano, Italy

Lorenza Lenzi, Pomarolo (TN), Italy

Choosing wisely Roberto Frediani, Chieri (TO), Italy

\section{PROGETTI SPECIALI}

AGGIORN@FADOI

Giuliano Pinna

PROGETTO NUOVE TECNOLOGIE

Francesco Nasso, Flavio Tangianu

PROGETTO MEDICINA DI GENERE

Cecilia Politi

PROGETTO COMPETENCE

Flavio Tangianu

PROGETTO GOVERNANCE

Stefano De Carli, Andrea Montagnani, Fabrizio Colombo

PROGETTO HOSPITALIST

Francesco Orlandini

PROGETTO FINE VITA

Mauro Carbone, Fabio Gilioli

PROGETTO GASTROENTEROLOGIA Q FEGATO

Luca Fontanella, Paola Piccolo, Franco Radaelli, Giancarlo Parisi

PROGETTO NUTRIZIONE CLINICA

Roberto Risicato, Luciano Tramontano

PROGETTO MALATTIE INFETTIVE/ANTIBIOTICI

Claudio Santini, Massimo Giusti, Marco Falcone

PROGETTO ECOGRAFIA INTERNISTICA

Francesco Cipollini, Nicola Mumoli

PROGETTO MALATTIE RARE

Antonio Brucato, Antonella Paradiso

PROGETTO BPCO/NIV

Marco Candela, Giuseppe De Matthaeis, Francesco Ventrella

PROGETTO TROMBOSI

Mauro Silingardi, Matteo Giorgi Pierfranceschi, Pierpaolo Di Micco PROGETTO TRIAL

Giancarlo Agnelli, Antonio Ceriello, Leo Fabbri, Claudio Ferri,

Franco Radaelli, Paolo Verdecchia

PRESIDENTE ANÍMO

Gabriella Bordin, Castelfranco Veneto (TV), Italy 


\section{QUADERNI - Italian Journal of Medicine}

Stipsi indotta da oppioidi: un problema emergente con nuove opportunità terapeutiche.

F. Radaelli, D. Di Paolo 


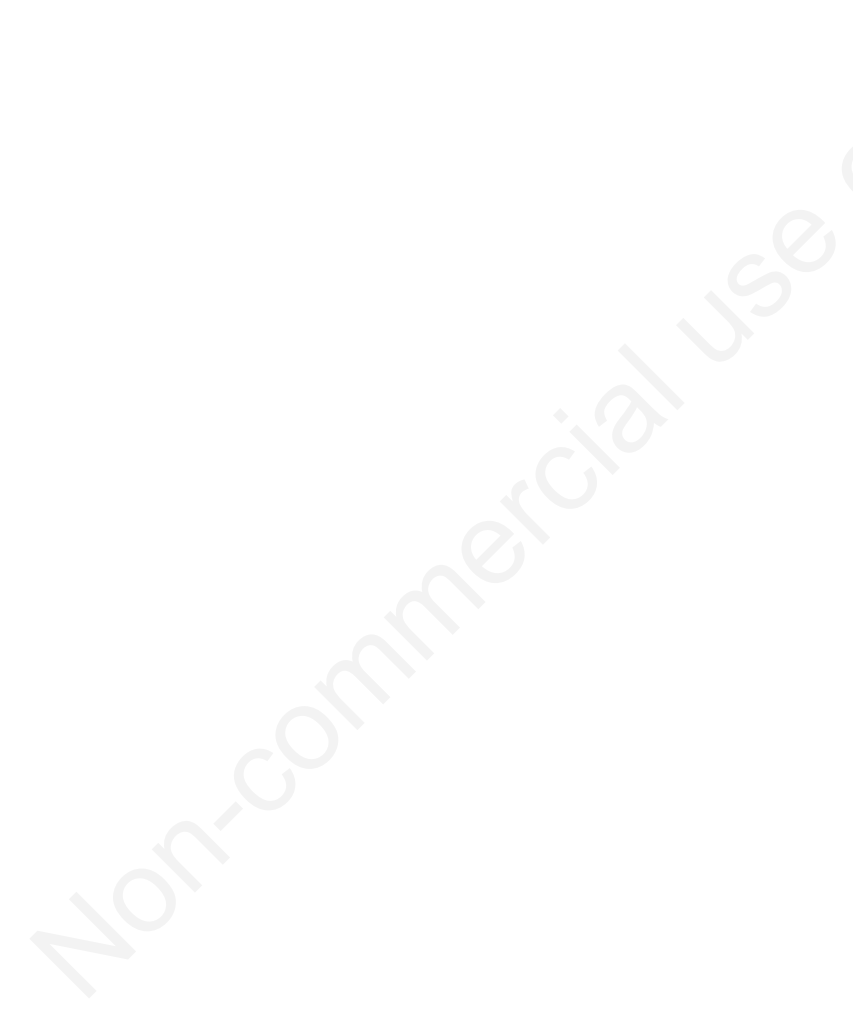




\title{
Stipsi indotta da oppioidi: un problema emergente con nuove opportunità terapeutiche
}

\author{
Franco Radaelli ${ }^{1}$, Dhanai Di Paolo ${ }^{1,2}$ \\ ${ }^{1}$ Unità Operativa Complessa di Gastroenterologia, Ospedale Valduce, Como; ${ }^{2}$ Fondazione IRCCS Ca' Granda Ospedale \\ Maggiore Policlinico, Milano, Italia
}

\section{Introduzione}

Gli analgesici oppioidi sono farmaci ampiamente utilizzati per il trattamento del dolore acuto e cronico di entità moderato-severa. Nelle ultime due decadi è stato osservato un progressivo incremento prescrittivo di questi farmaci, soprattutto per il controllo del dolore non oncologico ${ }^{1}$ e stime recenti indicano che circa il $4-5 \%$ della popolazione americana ne fa un regolare utilizzo. ${ }^{2}$ Anche in Italia, a partire dai primi anni dell'ultimo decennio, l'andamento temporale del consumo degli oppioidi ha mostrato un incremento percentualmente elevato, seppur inferiore in termini assoluti rispetto ad altri Paesi europei, principalmente determinato dal cambiamento della legislazione sulla prescrizione di questi farmaci (anno 2001) e, a seguire, dall'immissione in commercio di nuove formulazioni farmaceutiche e principi attivi.

Gli oppioidi agiscono ed esercitano la loro attività analgesica grazie al legame che si instaura fra queste molecole e specifici recettori - $\mu(\mathrm{mu}), \kappa($ kappa) e $\delta$ (delta) - presenti nel sistema nervoso centrale e periferico. La presenza di questi recettori anche nel tratto gastrointestinale - in particolare i recettori $\kappa$ nello stomaco e nel piccolo intestino e i recettori $\mu$ nel piccolo intestino e nel colon prossimale - rende ragione dei molteplici effetti avversi gastroenterici associati all'uso di questi farmaci (OIBD, Opioid Induced Bowel Dysfunction), che includono nausea, vomito, sintomi

Corrispondente: Franco Radaelli, Unità Operativa Complessa di Gastroenterologia, Ospedale Valduce, Via Dante 10, 22100 Como, Italia.

Tel.: +39.031324147 - Fax: +39.031324150 .

E-mail: francoradaelli01@gmail.com

Articolo pubblicato secondo la Creative Commons Attribution NonCommercial 4.0 License (CC BY-NC 4.0).

${ }^{\circ}$ Copyright: the Author(s), 2021

Licensee PAGEPress, Italy

QUADERNI - Italian Journal of Medicine 2021; 9(1):1-7 da reflusso gastroesofageo, meteorismo, distensione addominale, dolore addominale e stipsi. Nello specifico l'attivazione dei recettori enterici $\mu$ è responsabile della stipsi indotta da oppioidi (OIC, Opioid Induced Constipation), che rappresenta il più frequente effetto collaterale associato a questi farmaci ${ }^{3}$

Le stime della prevalenza della OIC riportata negli studi sono molto variabili. In una survey condotta in Europa e negli Stati Uniti su pazienti in trattamento con oppioidi orali per dolore neoplastico e non neoplastico (PROBE I, Prospective Randomized Open Blinded End-point), 1' $81 \%$ dei pazienti ha riferito un problema di stipsi; di questi, il $45 \%$ ha riportato una frequenza di meno di tre movimenti intestinali settimanali ed il 58\% una difficoltà nella espulsione delle feci. ${ }^{4}$ Studi osservazionali negli Stati Uniti suggeriscono una prevalenza di OIC in pazienti con dolore non neoplastico variabile tra il $40 \%$ e il $50 \%{ }^{5-7} \mathrm{La}$ OIC è problematica clinicamente rilevante, non solo per il suo impatto negativo sulla qualità di vita dei pazienti, ma anche per le ripercussioni negative sul trattamento del dolore. Diversi studi hanno infatti documentato che fino ad un terzo dei pazienti che sviluppano stipsi in corso di trattamento con oppioidi tende a ridurre la dose o interrompere la terapia analgesica nel tentativo di contrastare il problema, compromettendo di conseguenza il controllo del dolore, ${ }^{4,8}$

I meccanismi fisiopatologici della OIC sono complessi e principalmente attribuibili all'attivazione dei recettori enterici $\mu$ e alla conseguente inibizione del rilascio di neurotrasmettitori (acetilcolina). Questi recettori sono espressi principalmente nei neuroni del plesso mioenterico, che fisiologicamente controlla l'attività motoria propulsiva intestinale attraverso la contrazione ed il rilasciamento della muscolatura longitudinale e circolare della parete intestinale. Inoltre, i recettori $\mu$ sono presenti a livello dei neuroni del plesso sottomucoso, implicato nella secrezione e nell'assorbimento di fluidi. Gli oppioidi esercitano pertanto sui recettori $\mu$ tre principali effetti: i) inibizione della peristalsi con aumento delle contrazioni toniche non propulsive a livello del piccolo intestino e del colon; ii) aumento dell'assorbimento di fluidi a livello del colon, con aumento della 
consistenza delle feci; iii) riduzione della sensibilità rettale ed aumento del tono sfinteriale. La combinazione di questi effetti risulta in evacuazioni meno frequenti e più difficoltose. ${ }^{9}$

Considerate le sue peculiarità fisiopatologiche, oltre che di trattamento, la OIC è stata recentemente classificata come un'entità nosologica specifica. In accordo con i criteri classificativi di Roma IV dei disturbi gastrointestinali funzionali, la OIC viene definita come l'insorgenza de novo o il peggioramento di sintomi caratteristici della stipsi funzionale (riduzione dei movimenti spontanei dell'alvo, difficoltà nell'espulsione, senso di evacuazione incompleta, feci di consistenza aumentata) associati all'inizio o alla modifica di un trattamento cronico con oppioidi (Tabella 1). ${ }^{10}$

\section{Trattamento}

Recentemente molte società scientifiche hanno pubblicato linee guida sulla gestione della OIC, con lo scopo di sensibilizzare i clinici a questa problematica, nonché fornire indicazioni specifiche di trattamento basate sulle evidenze scientifiche ad oggi disponibili. ${ }^{11-17}$

In generale, tutte le linee guida raccomandano un approccio di trattamento step-up, che inizialmente preveda generiche indicazioni sullo stile di vita (in particolare un adeguato introito di fibre e di liquidi associato ad una regolare attività fisica moderata, qualora possibile) e l'utilizzo preventivo di lassativi tradizionali, osmotici o stimolanti. Le linee guida invitano inoltre a rivalutare la reale necessità di farmaci che possono contribuire ad un peggioramento della stipsi (ad esempio, antiacidi contenete alluminio, antidepressivi triciclic, antistaminici, antispastici, antipsicotici, supplementazioni di ferro e calcio, calcioanatagonisti), considerando eventuali alternative terapeutiche, e suggeriscono di valutare l'opportunità di switching a farmaci con minori effetti sulla motilità gastroenterica, come la combinazione di oppioidi con azione agonista/antagonista a livello recettoriale (es. ossicodone/naloxone) o formulazioni transdermiche.

Tabella 1. Definizione di Opioid Induced Constipation (stipsi indotta da oppioidi), in accordo con i criteri di Roma IV.

Insorgenza o peggioramento di sintomi all'inizio, alla modifica
o all'incremento posologico di un trattamento con oppioidi, con
due o più dei seguenti criteri, in $\geq 25 \%$ delle evacuazioni:

Sforzo al passaggio delle feci

Passaggio di feci di consistenza dura o grumosa

Sensazione di incompleta evacuazione

Necessità di manovre digitali per facilitare l'evacuazione

Meno di tre movimenti dell'alvo spontanei per settimana
I lassativi tradizionali osmotici (macrogol, lattulosio, sali di magnesio) o stimolanti (senna, bisacodile) rappresentano la prima di linea di trattamento alla luce della loro comprovata efficacia, dell'elevato profilo di sicurezza e del basso costo, ed il loro utilizzo dovrebbe essere considerato ogni qualvolta si inizi un trattamento con oppioidi. Meno razionale, considerando i meccanismi fisiopatologici della OIC, è invece l'uso di agenti formanti massa, il cui utilizzo pertanto non è consigliato se non in pazienti con evidente carenza di apporto di fibre con la dieta. È però corretto precisare che la maggior parte degli studi clinici finalizzati a valutare l'efficacia di questi lassativi hanno incluso pazienti con stipsi cronica non specificatamente attribuita all'uso di oppioidi. Sono infatti pochissimi gli studi clinici controllati che hanno valutato i lassativi tradizionali in questo specifico setting di pazienti. ${ }^{18-20}$ Nell'unico studio clinico randomizzato controllato (RCT) in doppio cieco ad oggi disponibile, condotto su 57 pazienti in terapia di mantenimento con metadone, i lassativi tradizionali (lattulosio e PEG) si sono dimostrati superiori rispetto a placebo nel migliorare la consistenza delle feci. Mancano tuttavia i dati sufficienti per effettuare un'analisi statistica e un confronto tra le diverse tipologie di lassativo in commercio. ${ }^{18}$ L'utilizzo add-on di supposte di glicerina o clismi a basso volume rappresentano un'ulteriore chance terapeutica in pazienti già in trattamento con lassativi osmotici e/o stimolanti. ${ }^{11}$

Nei pazienti refrattari al trattamento con comuni lassativi, le linee guida raccomandano l'utilizzo di farmaci specifici in grado di bloccare gli effetti degli oppioidi sui recettori enterici $\mu$ (PAMORAs, Periherally Acting $\mu$-Opioid Receptor Antagonist). Viceversa, non vi sono al momento evidenze sufficienti per considerare altre alternative farmacologiche per il trattamento della OIC, quali il lubiprostone, farmaco secretagogo in grado di incrementare la secrezione di acqua a livello intestinale attraverso un'azione sul canale del cloro, e la prucalopride, agonista selettivo del recettore 5-HT4 con azione procinetica sul colon. ${ }^{11}$

Caratteristica comune dei PAMORAs è l'azione selettiva sui recettori periferici $\mu$, in grado di contrastare gli effetti collaterali degli oppioidi a livello gastrointestinale, ristabilendo le funzioni fisiologiche del sistema nervoso enterico senza interferire con l'attività analgesica a livello del sistema nervoso centrale. ${ }^{21}$

Al fine di ottimizzare l'utilizzo di questi farmaci è fondamentale una corretta selezione dei pazienti refrattari ai trattamenti standard, preferibilmente attraverso l'utilizzo di strumenti validati. La Bowel Function Index (BFI) è la scala più comunemente adottata nella pratica clinica per la sua facilità di utilizzo. La BFI è caratterizzata da tre semplici domande che vengono poste al paziente durante la visita e che indagano, nei sette giorni precedenti la visita, tre parametri clinici: i) la facilità di 
evacuazione; ii) la sensazione di incompleta evacuazione; iii) il giudizio personale del paziente riguardo alla stipsi. Le risposte vengono valutate utilizzando una scala analogica (VAS, punteggio $0-100 ; 0=$ nessuna difficoltà; $100=$ grande difficoltà). Pazienti con uno score medio $\geq 30$ sono identificati come pazienti con un'inadeguata risposta al trattamento lassativo e come tali definiti refrattari. 22,23

Sono tre i PAMORAs attualmente in commercio: metilnaltrexone, naloxegol e naldemedina. Queste tre molecole hanno tutte dimostrato in RCTs una superiorità di efficacia rispetto a placebo verso end-point predefiniti, in genere rappresentati dall'aumento della frequenza delle evacuazioni (almeno 3 movimenti spontanei dell'alvo/settimana o più di 1 movimento dell'alvo/settimana rispetto al dato basale), dal miglioramento della consistenza delle feci e dalla riduzione dello sforzo e del dolore evacuativo. ${ }^{23-26}$ È però opportuno un accurato esame degli studi clinici per ciascuna molecola al fine di valutare correttamente il loro profilo di sicurezza ed efficacia, che dovrebbe poi guidare la scelta clinica.

\section{Naloxegol}

Il naloxegol è un derivato PEGilato del naloxone (antagonista del recettore $\mu$ ) che, a differenza di quest'ultimo, ha un effetto limitato sul sistema nervoso centrale grazie alla componente PEGilata della molecola che ne impedisce il passaggio attraverso la barriera emato-encefalica.

I dati a supporto dell'uso di naloxegol provengono da uno studio di fase $2^{27}$ e da due RTCs in doppio cieco di fase 3 (KODIAC-04 e KODIAC-05, Chey et $a l .{ }^{28}$ ) che confrontano l'efficacia di naloxegol $12.5 \mathrm{mg}$ o naloxegol $25 \mathrm{mg}$ rispetto a placebo in 1352 pazienti con OIC. I dati dei due RTCs mostrano un raggiungimento dell'end-point primario (almeno 3 movimenti intestinali per settimana) nel $42 \%$ dei pazienti trattati con naloxegol $25 \mathrm{mg}$ rispetto al $29 \%$ del braccio placebo (RR 1.43; 95\% CI, 1.19 to 1.71$)$. KODIAC-08 è un RTC multicentrico, in aperto, a gruppi paralleli (naloxegol $25 \mathrm{mg}$ vs usual care ed uso di lassativi standard) di fase 3 che ha valutato la sicurezza a lungo termine (a 52 settimane) del naloxegol in pazienti non oncologici con OIC. Il farmaco è stato considerato sicuro e ben tollerato, con insorgenza di effetti avversi di entità lieve-moderata (addominalgia, meteorismo, nausea, diarrea) simili nei due gruppi di trattamento (naloxegol $82 \%$ vs usual care $72 \%$ ). Nonostante la percentuale di eventi avversi osservata per naloxegol $25 \mathrm{mg}$ nello studio multicentrico sia superiore rispetto a quella riscontrata nei due RTCs di fase 3 (KODIAC04 61,2\%; KODIAC-05 69,0\%), il numero di eventi avversi che ha portato alla sospensione del farmaco è sovrapponibile nei tre studi (KODIAC-04, KODIAC$0510.3 \%$ vs KODIAC-08 10.5\%). ${ }^{29}$

Nella pratica clinica, il farmaco è utilizzato in mono-somministrazione orale al dosaggio di $25 \mathrm{mg}$, da assumere a stomaco vuoto almeno 30 minuti prima o 2 ore dopo il primo pasto del giorno. In pazienti con insufficienza renale di entità moderata o grave, la dose iniziale consigliata è di $12,5 \mathrm{mg}$, che può essere aumentata a $25 \mathrm{mg}$ in caso di buona tolleranza del paziente. Una dose iniziale ridotta del farmaco è, inoltre, suggerita nei pazienti in co-trattamento con inibitori moderati del CYP3A4 (es., verapamil, diltiazem).

È opportuno sottolineare che gli studi randomizzati sopra citati hanno incluso unicamente pazienti con dolore cronico non neoplastico, nonostante il farmaco sia approvato, per lo meno in Italia, con un'indicazione più generica per il trattamento della OIC con un'inadeguata risposta ai lassativi.

\section{Metilnaltrexone}

Il metilnaltrexone è stato il primo PAMORAs approvato dalla Food and Drug Administration (FDA) e dall'European Medicines Agency (EMA) per il trattamento della OIC in pazienti oncologici terminali (2008) e successivamente per il trattamento della OIC in pazienti con dolore non oncologico.

La molecola è disponibile in due formulazioni, per iniezioni sottocutanee e per uso orale (compresse). La sua struttura amminica quaternaria ne limita il passaggio attraverso la barriera emato-encefalica, permettendone un'azione esclusiva a livello periferico.

L'efficacia del metilnaltrexone è stata valutata in due RTCs di fase 3 in doppio cieco verso placebo comprendenti 722 pazienti non neoplastici con OIC. ${ }^{30,31} \mathrm{Gli}$ studi hanno confrontato l'efficacia del metilnaltrexone sottocutane $\mathrm{o}^{30} \mathrm{e}$ del metilnaltrexone orale ${ }^{31}$ rispetto a placebo per una durata di 4 settimane. Nel complesso, il $54 \%$ dei pazienti che hanno ricevuto metilnaltrexone ha raggiunto l'end-point primario (almeno 3 movimenti intestinali a settimana senza ricorrere all'utilizzo di rescue therapy) rispetto al $38 \%$ dei pazienti del braccio placebo (RR 1,43; IC 95\%, 1,21-1,68). Un successivo RTC multicentrico, in aperto, di fase 3 ha valutato la sicurezza $\mathrm{e}$ la tollerabilità del metilnaltrexone sottocutaneo $(12 \mathrm{mg} / \mathrm{die})$ in 1034 pazienti con dolore cronico non neoplastico con OIC, con un follow-up di 48 settimane. Il farmaco si è mostrato efficace in termini di miglioramento del numero e della consistenza delle evacuazioni rispetto al basale (ma l'uso concomitante di lassativi è stato segnalato dal $77 \%$ dei pazienti) e in media ben tollerato, con insorgenza di effetti avversi tali da giustificare la sospensione del farmaco nel $15 \%$ dei pazienti (totale dei soggetti con effetti avversi: $79 \%$ ). ${ }^{18}$

Il farmaco è disponibile per uso clinico come formulazione sottocutanea $(8 \mathrm{mg} / 0,4 \mathrm{~mL} ; 12 \mathrm{mg} / 0,6 \mathrm{~mL})$ o compresse orali $(150 \mathrm{mg})$. La dose raccomandata per la OIC in pazienti adulti con dolore cronico (con esclusione dei pazienti sottoposti a cure palliative con malattia avanzata) è di $12 \mathrm{mg}$, somministrata in dose 
singola a giorni alterni (4 dosi a settimana) o di $450 \mathrm{mg}$ per os. In pazienti con malattia avanzata (pazienti sottoposti a cure palliative), il regime raccomandato è sottocute, con una dose di $8 \mathrm{mg}$ (pazienti il cui peso sia compreso tra 38 e $61 \mathrm{~kg}$ ) o di $12 \mathrm{mg}$ (pazienti il cui peso sia compreso tra 62 e $114 \mathrm{~kg}$ ), con somministrazione in dose singola a giorni alterni. In base alle esigenze cliniche possono essere somministrate dosi ad intervalli più lunghi. L'utilizzo di due dosi consecutive a distanza di 24 ore è indicato solo nel caso in cui non ci sia stata risposta (movimento intestinale) alla dose del giorno precedente. Pazienti il cui peso sia al di fuori dei limiti devono ricevere una dose pari a $0,15 \mathrm{mg} / \mathrm{kg}$ del loro peso. Aggiustamenti posologici sono richiesti in caso di insufficienza renale moderata $(\mathrm{CrCl}<60 \mathrm{~mL} / \mathrm{min})$ o di grave alterazione della funzione epatica. Rispetto agli altri PAMORAs, il metilnaltrexone non viene metabolizzato dal sistema CYP3A4, e come tale ha scarse interazioni farmacologiche.

\section{Naldemedina}

La naldemedina rappresenta l'ultima delle molecole approvate dalla FDA ed EMA per il trattamento della OIC, rispettivamente nel 2017 e nel 2019. Dal punto di vista chimico è un derivato del naltrexone e, come questa molecola, blocca i recettori degli oppioidi $\mu, \delta$, e k. Mentre il naltrexone è in grado di attraversare la barriera emato-encefalica, la catena laterale idrofila della naldemedina e la sua affinità con la P-glicoproteina determinano concentrazioni trascurabili della molecola nel sistema nervoso centrale e una conseguente azione periferica selettiva sui recettori del tratto gastrointestinale. ${ }^{32,33} \mathrm{Il}$ farmaco è principalmente metabolizzato in un derivato inattivo dal citocromo CYP3A4. Pertanto, l'uso concomitante di naldemedina con inibitori forti del CYP3A4 (ad es., succo di pompelmo, itroconazolo, ketoconazolo, ritonavir, claritromicina) comporta un aumento dell'esposizione alla naldemedina che può potenzialmente aumentare il rischio di reazioni avverse (es. sindrome da astinenza da oppioidi). Viceversa, l'uso concomitante del farmaco con induttori forti del CYP3A4 (ad es. rifampicina, carbamazepina, fenobarbital e fenitoina) può provocare una diminuzione dell'esposizione alla naldemedina, con possibile riduzione della sua efficacia.

I dati a supporto dell'uso della naldemedina provengono da 4 RCTs in doppio cieco verso placebo, tra cui uno studio di fase $2 \mathrm{~b}^{34} \mathrm{e}$ tre studi di fase 3 (COMPOSE 1,2 e 3), ${ }^{35,36}$ per un totale di più di 2400 pazienti inclusi. L'end-point principale per tre di questi studi ${ }^{34,35}$ è la capacità di ottenere almeno 3 movimenti intestinali spontanei a settimana. Circa il $52 \%$ dei pazienti trattati con naldemedina ha raggiunto l'end-point primario rispetto al 35\% di quelli trattati con placebo (RR 1,51; 95\% CI, 1,32 a 1,72). Lo studio COMPOSE 3 ha valutato la sicurezza a lungo termine della naldemedina in pazienti con OIC e dolore cronico non oncologico in 52 settimane di follow-up. ${ }^{37}$ L'utilizzo del farmaco è stato associato ad un aumento di circa un movimento spontaneo di feci a settimana rispetto a placebo, con un miglioramento statisticamente significativo della consistenza delle feci, dello sforzo evacuativo e della qualità della vita. Sebbene gli eventi avversi (principalmente dolore addominale, diarrea, flatulenza, nausea, mal di schiena ed infezioni) che hanno portato all'interruzione del trattamento nei 4 studi sono più comuni nel braccio di trattamento (RR 1.44; 95\% CI, 1.03-2.03), è doveroso sottolineare che l'aumento assoluto degli eventi avversi è basso ( 2 eventi avversi in più per 100 pazienti trattati rispetto a placebo) e pertanto da considerarsi clinicamente poco rilevante. Una recente metanalisi di 5 RCTs conferma la maggiore incidenza di eventi avversi nel braccio di trattamento con naldemedina rispetto a placebo, sottolineando però come gli eventi riportati siano tutti di entità lieve-moderata e ben tollerati dai soggetti trattati. ${ }^{37}$ Gli studi COMPOSE 4 e COMPOSE 5 hanno valutato l'utilizzo della naldemedina in pazienti oncologici. ${ }^{38} \mathrm{Nel} \mathrm{COM}-$ POSE 4, 193 pazienti con OIC già in trattamento con lassativi sono stati randomizzati a naldemedina o placebo per un periodo di studio di 2 settimane, con endpoint primario l'efficacia del farmaco. Nel COMPOSE 5 , i pazienti che hanno ricevuto naldemedina sono stati seguiti in aperto per un periodo di 12 settimane, con lo scopo primario di valutare la sicurezza del farmaco. I due studi hanno sostanzialmente confermato i risultati di efficacia e sicurezza già dimostrati dagli studi condotti in pazienti non oncologici.

Nella pratica clinica il farmaco è disponibile nella formulazione orale, in compresse da 200 microgrammi da assumere in mono-somministrazione, senza necessità di modifiche posologiche né nei pazienti con insufficienza renale né nei pazienti con compromissione lieve/moderata della funzionalità epatica. Una cautela nell'utilizzo della naldemedina va considerata, invece, nei pazienti che assumo inibitori o induttori del CYP3A4 e nei pazienti con alterazioni della barriera emato-encefalica (ad es. tumori maligni primitivi cerebrali, metastasi, sclerosi multipla in fase attiva o altre condizioni infiammatorie a carico del sistema nervoso centrale), in cui maggiore è il rischio di astinenza da oppioidi o di ridotta analgesia.

\section{Le linee guida dell'American Gastroenterology Association sul trattamento della OIC e sull'utilizzo dei PAMORAs}

L'American Gastroenterology Association (AGA) ha recentemente valutato criticamente, mediante metodologia GRADE (Grading of Recommendations Assessment, Development and Evaluation), i dati di sicurezza 
Tabella 2. Riassunto delle raccomandazioni delle linee guida dell'American Gastroenterological Association (AGA) per la gestione della stipsi indotta da oppioidi.

\begin{tabular}{|c|c|c|}
\hline Raccomandazioni & $\begin{array}{l}\text { Forza della } \\
\text { raccomandazione }\end{array}$ & $\begin{array}{l}\text { Qualità della } \\
\text { evidenza }\end{array}$ \\
\hline $\begin{array}{l}\text { 1. Lassativi tradizionali } \\
\text { a. In pazienti con OIC, l'AGA raccomanda l'utilizzo di lassativi come agenti di prima linea }\end{array}$ & Forte & Moderata \\
\hline $\begin{array}{l}\text { 2. PAMORAs } \\
\text { a. In pazienti con OIC refrattaria all'uso di lassativi, l'AGA raccomanda l'uso di naldemedina } \\
\text { versus nessun trattamento }\end{array}$ & Forte & Elevata \\
\hline $\begin{array}{l}\text { b. In pazienti con OIC refrattaria all'uso di lassativi, l'AGA raccomanda l'uso di naloxegol } \\
\text { versus nessun trattamento } \\
\text { c. In pazienti con OIC refrattaria all'uso di lassativi, l'AGA raccomanda l'uso di metilnaltrexone } \\
\text { versus nessun trattamento }\end{array}$ & Condizionale & Moderata \\
\hline $\begin{array}{l}\text { 3. Segretagoghi intestinali } \\
\text { a. In pazienti con OIC, l'AGA non esprime alcuna raccomandazione sull'uso di lubiprostone }\end{array}$ & Nessuna raccomandazione & Insufficiente \\
\hline $\begin{array}{l}\text { 4. Agonisti selettivi 5-HT } \\
\text { a. In pazienti con OIC, l'AGA non esprime alcuna raccomandazione sull'uso di prucalopride }\end{array}$ & Nessuna raccomandazione & Insufficiente \\
\hline
\end{tabular}

e di efficacia dei trattamenti farmacologici della OIC, formulando raccomandazioni sul loro utilizzo nella pratica clinica. ${ }^{11,24}$ Le principali raccomandazioni sono riassunte nella Tabella 2. Queste includono l'utilizzo dei lassativi tradizionali come prima linea di trattamento e l'utilizzo dei PAMORAs nei pazienti refrattari con controllo subottimale della stipsi, in analogia a quanto proposto da altre linee guida.

Le linee guida AGA riconoscono alla naldemedina prove di efficacia e sicurezza superiori rispetto a naloxegol e metilnaltrexone, pur in assenza di studi di confronto diretto tra i differenti PAMORAs. La disponibilità di studi condotti sia nei pazienti oncologici che non-oncologici e di dati solidi di efficacia e sicurezza, anche a lungo termine, sono sicuramente un punto a favore di questa molecola. È per questo che la naldemedina è l'unica molecola tra i PAMORAs a ricevere una raccomandazione forte al suo utilizzo nelle linee guida dell'AGA, che pertanto suggeriscono in modo indiretto il suo utilizzo preferenziale rispetto alle altre molecole della stessa classe. Il giudizio delle linee guida sul metilnaltrexone è invece complessivamente poco favorevole, per l'eterogeneità e l'imprecisione dei dati a disposizione, pur riconoscendo alla molecola possibili vantaggi in un sottogruppo di pazienti (ad esempio quelli con vomito ricorrente) che potrebbero beneficiare della somministrazione sottocutanea.

\section{TAKE HOME MESSAGES}

- Il progressivo incremento prescrittivo degli oppioidi, soprattutto per il controllo del dolore non oncologico, ha reso indispensabile la sensibilizzazione dei clinici alla gestione degli effetti avversi gastroenterici associati all'uso di questi farmaci, in particolare della stipsi.

- I farmaci specifici in grado di bloccare selettivamente gli effetti degli oppioidi sui recettori enterici $\mu(P A$ MORAs, Periherally Acting $\mu$-Opioid Receptor Antagonist) rappresentano l'opzione terapeutica di scelta nei pazienti con stipsi indotta da oppioidi refrattari al trattamento con comuni lassativi.

- Studi clinici randomizzati hanno dimostrato per tutti e ter i PAMORAs attualmente in commercio (metilnaltrexone, naloxegol e naldemedina) una superiorità di efficacia rispetto a placebo verso end-point predefiniti (aumento della frequenza delle evacuazioni, miglioramento della consistenza delle feci e riduzione dello sforzo e del dolore evacuativo).

- Le naldemedina presentano dati di efficacia e sicurezza superiori rispetto a naloxegol e metilnaltrexone, essendo il farmaco stato valutato, anche con follow-up a lungo termine, sia in pazienti con dolore oncologico che non-oncologico. Pertanto, le linee guida dell'American Gastroenterology Association forniscono una raccomandazione forte al suo utilizzo, suggerendo indirettamente il suo uso preferenziale rispetto alle altre molecole della stessa classe. 


\section{Bibliografia}

1. Sullivan MD, Edlund MJ, Fan MY, et al. Trends in use of opioids for non-cancer pain conditions 2000-2005 in commercial and Medicaid insurance plans: the TROUP study. Pain 2008;138:440-9.

2. Sullivan MD, Edlund MJ, Zhang L, et al. Association between mental health disorders, problem drug use, and regular prescription opioid use. Arch Intern Med 2006; 166:2087-93.

3. Farmer AD, Drewes AM, Chiarioni G, et al. Pathophysiology and management of opioid-induced constipation: European expert consensus statement. United Eur Gastroenterol J 2019;7:7-20.

4. Bell TJ, Panchal SJ, Miaskowski C, et al. The prevalence, severity, and impact of opioid-induced bowel dysfunction: results of a US and European patient survey (PROBE 1). Pain Med 2009;10:35-42.

5. Panchal SJ, Muller-Schwefe P, Wurzelmann JI. Opioidinduced bowel dysfunction: prevalence, pathophysiology and burden. Int J Clin Pract 2007;61:1181-7.

6. Brown RT, Zuelsdorff M, Fleming M. Adverse effects and cognitive function among primary care patients taking opioids for chronic nonmalignant pain. J Opioid Manag 2006;2:137-46.

7. Cook SF, Lanza L, Zhou X, et al. Gastrointestinal side effects in chronic opioid users: results from a populationbased survey. Aliment Pharmacol Ther 2008;27:122432.

8. Streicher JM, Bilsky EJ. Peripherally acting $\mu$-opioid receptor antagonists for the treatment of opioid-related side effects: mechanism of action and clinical implications. J Pharmacy Pract 2018;31:658-69.

9. Pannemans J, Vanuytsel T, Tack J. New developments in the treatment of opioid-induced gastrointestinal symptoms. United Eur Gastroenterol J 2018;6:1126-35.

10. Drossman DA, Chang L, Chey WD, et al. Rome IV functional gastrointestinal disorders - disorders of gut-brain interaction. I. Raleigh, NC: The Rome Foundation; 2016.

11. Crockett SD, Greer KB, Heidelbaugh JJ, et al. American Gastroenterological Association Institute Guideline on the medical management of opioid-induced constipation. Gastroenterology 2019;156:218-26.

12. Larkin PJ, Cherny NI, La Carpia D, et al. ESMO Guidelines Committee. Diagnosis, assessment and management of constipation in advanced cancer: ESMO Clinical Practice Guidelines. Ann Oncol 2018;29:iv111iv125.

13. Davies A, Leach C, Caponero R, et al. MASCC recommendations on the management of constipation in patients with advanced cancer. Support Care Cancer 2020;28:23-33.

14. Müller-Lissner S, Bassotti G, Coffin B, et al. Opioidinduced constipation and bowel dysfunction: a clinical guideline. Pain Med 2017;18:1837-63.

15. Rogers B, Ginex PK, Anbari A, et al. ONS Guidelines ${ }^{\mathrm{TM}}$ for opioid-induced and non-opioid-related cancer constipation. Oncol Nurs Forum 2020;47:671-91.

16. Argoff CE, Brennan MJ, Camilleri M, et al. Consensus recommendations on initiating prescription therapies for opioid-induced constipation. Pain Med 2015;16:2324-37.

17. Drewes AM, Munkholm P, Simrén M, et al. Definition, diagnosis and treatment strategies for opioid-induced bowel dysfunction-Recommendations of the Nordic Working Group. Scand J Pain 2016;11:111-22.

18. Freedman MD, Schwartz HJ, Roby R, et al. Tolerance and efficacy of polyethylene glycol 3350/electrolyte solution versus lactulose in relieving opiate induced constipation: a double-blinded placebo-controlled trial. J Clin Pharmacol 1997;37:904-7.

19. Twycross RG, McNamara P, Schuijt C, et al. Sodium picosulfate in opioid-induced constipation: results of an open-label, prospective, dose-ranging study. Palliat Med 2006;20:419-23.

20. Wirz S, Nadstawek J, Elsen C, et al. Laxative management in ambulatory cancer patients on opioid therapy: a prospective, open-label investigation of polyethylene glycol, sodium picosulphate and lactulose. Eur J Cancer Care 2012;21:131-40.

21. Burr NE, Ford AC. Assessing the efficacy of peripherally acting $\mu$-opioid receptor antagonists (PAMORAs) in the treatment of opioid-induced constipation: authors reply. Gut 2019;68:1530-1.

22. Rentz AM, Yu R, Muller-Lissner S, et al. Validation of the Bowel Function Index to detect clinically meaningful changes in opioid-induced constipation. J Med Econ 2009;12:371-83.

23. Ueberall MA, Muller-Lissner S, Buschmann-Kramm C, et al. The Bowel Function Index for evaluating constipation in pain patients: definition of a reference range for a non-constipated population of pain patients. J Int Med Res 2011;39:41-50.

24. Hanson B, Siddique SM, Scarlett Y, et al. American Gastroenterological Association Institute Clinical Guidelines Committee. American Gastroenterological Association Institute technical review on the medical management of opioid-induced constipation. Gastroenterology 2019;156:229-253.e5.

25. Nee J, Zakari M, Sugarman MA, et al. Efficacy of treatments for opioid-induced constipation: systematic review and meta-analysis. Clin Gastroenterol Hepatol 2018;16:1569-84.

26. Vijayvargiya $\mathrm{P}$, Camilleri M, Vijayvargiya $\mathrm{P}$, et al. Systematic review with meta-analysis: efficacy and safety of treatments for opioid-induced constipation. Aliment Pharmacol Ther 2020;52:37-53.

27. Webster L, Dhar S, Eldon M, et al. A phase 2, doubleblind, randomized, placebo-controlled, dose-escalation study to evaluate the efficacy, safety, and tolerability of naloxegol in patients with opioid-induced constipation. Pain 2013;154:1542-50.

28. Chey WD, Webster L, Sostek M, et al. Naloxegol for opioid-induced constipation in patients with noncancer pain. N Engl J Med 2014;370:2387-96.

29. Webster L, Chey WD, Tack J, et al. Randomised clinical trial: the long-term safety and tolerability of naloxegol in patients with pain and opioid-induced constipation. Aliment Pharmacol Ther 2014;40:771-9.

30. Michna E, Blonsky ER, Schulman S, et al. Subcutaneous methylnaltrexone for treatment of opioidinduced constipation in patients with chronic, nonmalignant pain: a randomized controlled study. J Pain 2011;12:554-62.

31. Rauck R, Slatkin NE, Stambler N, et al. Randomized, Double-blind trial of oral methylnaltrexone for the treatment of opioid-induced constipation in patients with 
chronic noncancer pain. Pain Pract 2016;17:820-8.

32. Webster L, Michna E, Khan A, et al. Long-term safety and efficacy of subcutaneous methylnaltrexone in patients with opioid-induced constipation and chronic noncancer pain: a phase 3, open-label trial. Pain Med 2017;18:1496-504.

33. Coluzzi F, Scerpa MS, Pergolizzi J. Naldemedine: a new option for OIBD. J Pain Res 2020;13:1209-22.

34. Webster LR, Nalamachu S, Morlion B, et al. Long-term use of naldemedine in the treatment of opioid-induced constipation in patients with chronic noncancer pain: a randomized, double-blind, placebo-controlled phase 3 study. Pain 2018;159:987-94.

35. Hale M, Wild J, Reddy J, et al. Naldemedine versus placebo for opioid-induced constipation (COMPOSE-1 and COMPOSE-2): two multicentre, phase 3, doubleblind, randomised, parallel-group trials. Lancet Gastroenterol Hepatol 2017;2:555-64.

36. Webster LR, Yamada T, Arjona Ferreira JC. A phase 2b, randomized, double-blind placebo-controlled study to evaluate the efficacy and safety of naldemedine for the treatment of opioid-induced constipation in patients with chronic noncancer pain. Pain Med 2017;18:2350-60.

37. Song X, Wang D, Qu X, et al. A meta-analysis of naldemedine for the treatment of opioid-induced constipation. Expert Rev Clin Pharmacol 2019;12:121-8.

38. Katakami N, Harada T, Murata T, et al. Randomized phase III and extension studies of naldemedine in patients with opioid-induced constipation and cancer. J Clin Oncol 2017;35:3859-66. 


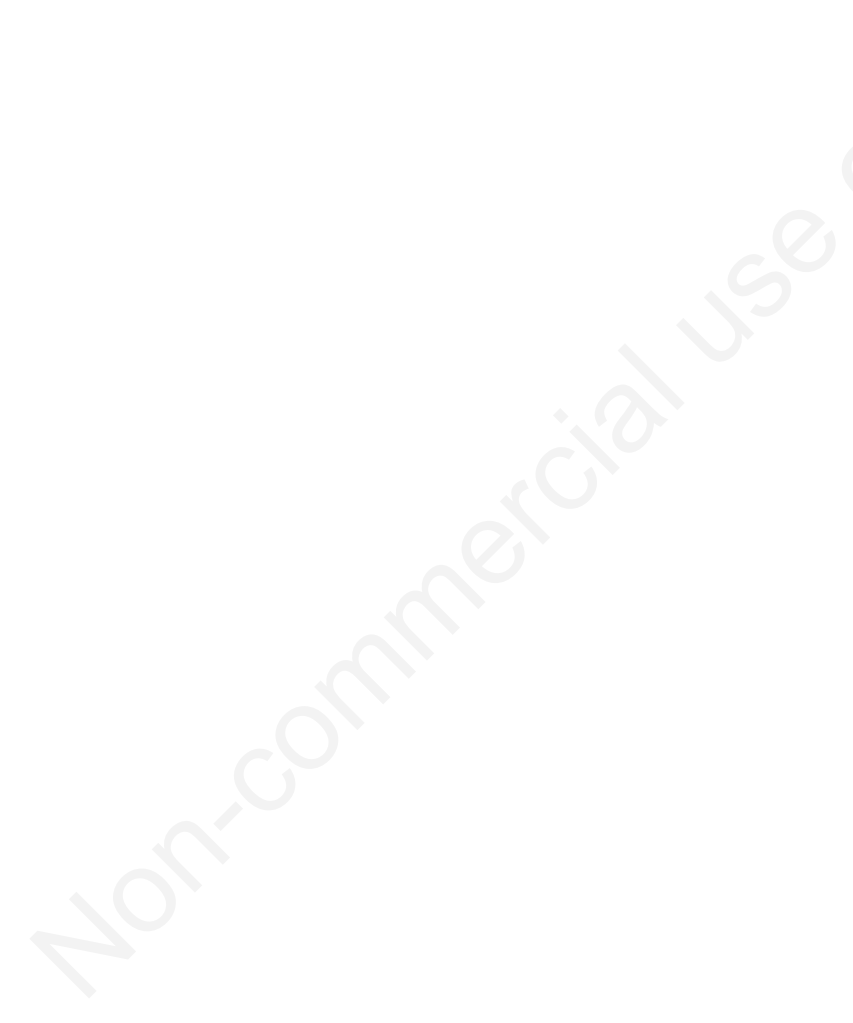




\section{LINEE GUIDA PER GLI AUTORI}

I Quaderni dell'Italian Journal of Medicine (Quaderni ITJM), costituiscono una collana supplementare solo online annessa alla rivista Italian Journal of Medicine contenente lavori solo in lingua italiana.

I Quaderni ITJM pubblicano:

- Monografie ad hoc individuate dal Presidente FADOI, dal Consiglio Direttivo, dal Board Scientifico o dall'Editor in Chief dell'Italian Journal of Medicine, in funzione del contesto scientifico-istituzionale attuale.

- Monografie ad hoc su temi di particolare rilevanza scientifica a cura della Commissione FADOI Giovani.

- Traduzioni in italiano di alcuni lavori pubblicati sui numeri standard dell'Italian Journal of Medicine, di particolare interesse per la comunità scientifica.

\section{STESURA DEI LAVORI}

I lavori dovranno essere redatti in modo conforme alle linee guida sotto riportate:

- I manoscritti devono essere scritti interamente in lingua italiana, su documento di Word, con carattere Times New Roman/Arial, dimensione 12, formato A4, interlinea doppia e margini 2,54 cm.

Parole totali: $\max 4000$; Sommario/Abstract: $\max 250$ parole; Bibliografia: $\min 40$ voci; Tabelle e Figure: $3 / 5$ totali (le tabelle non devono superare n. 1 pagina del documento in Word).

- La strutturazione del contenuto deve attenersi agli standard internazionali per la Rassegna (Review): i) Abstract riassuntivo dell'intero lavoro; ii) Introduzione al tema trattato; iii) Criteri e strumenti di ricerca (criteri di inclusione/esclusione, banche dati consultate, ...); iv) i successivi paragrafi devono illustrare le più recenti scoperte scientifiche nel settore; v) Conclusioni; vi) Bibliografia.

- La prima pagina deve riportare: i) titolo (in stampatello minuscolo), senza acronimi; ii) nome e cognome per esteso di ciascun autore; iii) affiliazione(i) di ciascun autore, numerate con numeri arabi; iv) eventuali ringraziamenti; v) nome e indirizzo postale completi dell'autore corrispondente, corredati da telefono, fax, e-mail; vi) da 3 a 5 parole chiave, separate da virgola. La seconda pagina può riportare: i) contributi degli autori, e.g. informazioni relative a contributi sostanziali delle persone coinvolte nello studio (http://www.icmje.org/\#author); ii) dichiarazione relativa a potenziali conflitti d'interesse; iii) ulteriori informazioni (e.g. fondi, esposizioni durante conferenze...).

- In caso di utilizzo di tabelle, queste devono essere tutte numerate con numeri arabi e citate nel testo in ordine consecutivo (e.g. NON nominare le tabelle come Tabella $1 \mathrm{~A}, 1 \mathrm{~B}, \ldots$ o $1.0,1.1 \ldots$. Le tabelle devono essere presentate in formato editabile. Ciascuna tabella deve essere corredata da una breve didascalia; in caso di abbreviazioni, riportare una nota a piè di CIASCUNA tabella che spieghi TUTTE le abbreviazioni presenti in ognuna.

- In caso di utilizzo di figure, queste devono essere inviate in formato tiff o .jpg, allegate al manoscritto in singoli files, secondo le seguenti specifiche:

i) a colori (salvate in modalità CMYK): minimo 300 dpi di risoluzione;

ii) in bianco e nero: minimo 600 dpi di risoluzione;

iii) minimo $17,5 \mathrm{~cm}$ di larghezza.

Ciascuna figura deve essere corredata da una breve didascalia.

NB: In caso di Tabelle/Figure riprese e/o modificate da altri lavori già pubblicati, sarà cura degli autori accertarsi se tali materiali siano o meno coperti da copyright e procurarsi i permessi necessari per la riproduzione. Tali permessi dovranno essere allegati alla versione definitiva del lavoro. L'ufficio editoriale si riserva la facoltà di rimuovere Tabelle/Figure coperte da copyright, se sprovviste dei necessari permessi.

- In caso di utilizzo di abbreviazioni, la prima volta che esse sono citate è necessario scrivere per esteso la definizione+abbreviazione tra parentesi tonde [e.g. risonanza magnetica $(\mathrm{RMN})]$, a seguire si dovrà riportare solo l'abbreviazione (unica eccezione: nei titoli e nelle didascalie di tabelle e figure NON si utilizzano abbreviazioni).

\section{BIBLIOGRAFIA}

Le voci bibliografiche devono essere formattate secondo lo stile Vancouver.

Nella sezione Bibliografia, le voci bibliografiche devono essere numerate consecutivamente nell'ordine in cui appaiono per la prima volta nel testo (NON in ordine alfabetico) e, nel testo, devono essere indicate con numeri arabi in apice. Voci bibliografiche riferite a comunicazioni personali o dati non pubblicati devono essere incorporate nel testo e NON inserite tra le voci numerate [e.g. (Wright 2011, dati non pubblicati) o (Wright 2011, comunicazione personale)]. Le voci bibliografiche nella sezione Bibliografia devono tassativamente essere preparate come segue:

i) più di 3 autori, citare 3 autori, et al. Se il lavoro contiene solo 4 autori, citarli tutti e 4 ;

ii) titolo del lavoro in stampatello minuscolo;

iii) nome della rivista, senza punti, abbreviato secondo gli standard internazionali; in caso di dubbi sulla corretta abbreviazione, fare riferimento ai seguenti siti:

a. ISI Journal Abbreviations Index (http://library.caltech.edu/ reference/abbreviations/);

b. Biological Journals and Abbreviations (http://home.ncifcrf. gov/research/bja/);

c. Medline List of Journal Titles (ftp://ftp.ncbi.nih.gov/pubmed/J_Medline.txt);

iv) inserire l'anno di pubblicazione subito dopo il nome della rivista, seguito da punto e virgola;

v) NON inserire giorno o mese di pubblicazione;

vi) citare solo il volume, seguito dai due punti (NON citare il fascicolo tra parentesi);

vii) abbreviare le pagine, e.g. 351-8.

Per accertarsi di aver correttamente formattato le voci bibliografiche, confrontarle con le citazioni in PubMed (http://www.ncbi. nlm.nih.gov/pubmed).

Esempi (prestare attenzione anche alla punteggiatura):

Articolo standard su Rivista

Halpern SD, Ubel PA, Caplan AL. Solid-organ transplantation in HIV-infected patients. N Engl J Med 2002;347:284-7.

\section{Proceedings}

Christensen S, Oppacher F. An analysis of Koza's computational effort statistic for genetic programming. In: Foster JA, Lutton E, Miller J, Ryan C, Tettamanzi AG, eds. Genetic programming. EuroGP 2002: Proceedings of the 5th European Conference on Genetic Programming, 2002 Apr 3-5, Kinsdale, Ireland. Berlin: Springer; 2002. pp 182-91.

Articoli i cui autori sono Organizzazioni

Diabetes Prevention Program Research Group. Hypertension, insulin, and proinsulin in participants with impaired glucose tolerance. Hypertension 2002;40:679-86.

Libri

Murray PR, Rosenthal KS, Kobayashi GS, Pfaller MA. Medical microbiology. 4th ed. St. Louis, MO: Mosby; 2002. (CITAZIONE DEL LIBRO INTERO)

Meltzer PS, Kallioniemi A, Trent JM. Chromosome alterations in human solid tumors. In: Vogelstein B, Kinzler KW, eds. The genetic basis of human cancer. New York, NY: McGraw-Hill; 2002. pp 93113. (CITAZIONE DI UN CAPITOLO)

\section{MODALITÀ D'INVIO DEI LAVORI}

Monografie

Gli Autori dovranno fare riferimento all'Editor-in-Chief o alle persone da lui designate nelle lettere di invito a scrivere gli articoli programmati.

Per gli articoli inviati da giovani internisti, gli Autori dovranno fare riferimento al Dr. Michele Meschi (e-mail: mmeschi@ausl.pr.it) e agli eventuali altri referenti da lei designati, nelle prime fasi di stesura dei manoscritti, revisioni e correzioni. 


\section{QUADERNI}

dell'Italian Journal

of Medicine

Il Dr. Meschi raccoglierà poi le versioni definitive dei lavori di ciascuna monografia e provvederà all'invio di tutti i materiali all'ufficio editoriale.

I lavori solo nella loro versione definitiva e approvata dalla Commissione FADOI Giovani dovranno pervenire all'ufficio editoriale già pronti per l'impaginazione e immediata pubblicazione (già corredati da eventuali permessi per la riproduzione di tabelle e immagini redatti secondo le presenti linee guida).

\section{Traduzioni}

Previo invito dell'Editor-in-Chief, gli Autori dovranno far pervenire all'ufficio editoriale la versione tradotta in italiano, al seguente indirizzo e-mail: paola.granata@pagepress.org

Il file in formato Word dovrà essere formattato secondo gli standard editoriali della rivista ufficiale ed essere già pronto per impagina- zione e immediata pubblicazione (corredato da eventuali permessi per la riproduzione di tabelle e immagini).

Si prega di inviare le eventuali tabelle in formato editabile e le figure in alta definizione secondo gli standard sopra riportati.

\section{NOTA PER GLI AUTORI}

I lavori pubblicati sui Quaderni ITJM non verranno indicizzati, ma saranno liberamente disponibili in un'apposita sezione del sito FADOI (http://www.fadoi.org/) e della rivista ufficiale.

Gli Autori i cui lavori siano accettati per la pubblicazione sui Quaderni ITJM e che fossero interessati a vederli pubblicati anche sulla rivista ufficiale, dovranno sottomettere attraverso il sito dell'ITJM (www.italjmed.org) la versione (già tradotta) in inglese e redatta in modo conforme alle linee guida della rivista; seguiranno poi la procedura di selezione tramite peer review e, se accettati, saranno inseriti nel piano editoriale standard.

\section{STAFF EDITORIALE}

Paola Granata, Journal Manager paola.granata@pagepress.org

Claudia Castellano, Production Editor

Tiziano Taccini, Technical Support

\section{PUBBLICATO DA}

PAGEPress Publications

via A. Cavagna Sangiuliani 5

27100 Pavia, Italy

T. +39.0382 .1549020$

F: +39.0382 .172745$

\section{pagepress}

www.pagepress.org info@pagepress.org

\section{QUADERNI - ITALIAN JOURNAL OF MEDICINE}

Tutti gli articoli pubblicati sui QUADERNI - Italian Journal of Medicine sono redatti sotto la responsabilità degli Autori. La pubblicazione o la ristampa degli articoli della rivista deve essere autorizzata per iscritto dall'editore. Ai sensi dell'art. 13 del D.Lgs 196/03, i dati di tutti i lettori saranno trattati sia manualmente, sia con strumenti informatici e saranno utilizzati per l'invio di questa e di altre pubblicazioni e di materiale informativo e promozionale. Le modalità di trattamento saranno conformi a quanto previsto dall'art. 11 del D.Lgs 196/03. I dati potranno essere comunicati a soggetti con i quali PAGEPress intrattiene rapporti contrattuali necessari per l'invio delle copie della rivista. Il titolare del trattamento dei dati è PAGEPress Srl, via A. Cavagna Sangiuliani 5 - 27100 Pavia, al quale il lettore si potrà rivolgere per chiedere l'aggiornamento, l'integrazione, la cancellazione e ogni altra operazione di cui all'art. 7 del D.Lgs 196/03.

https://www.italjmed.org/index.php/ijm/quad 
$\boldsymbol{\nabla}$ Medicinale sottoposto a monitoraggio addizionale. Ciò permetterà la rapida identificazione di nuove informazioni sulla sicurezza. Agli operatori sanitari è richiesto di segnalare qualsiasi reazione avversa sospetta. Vedere paragrafo 4.8 per informazioni sulle modalità di segnalazione delle reazioni avverse.

\section{DENOMINAZIONE DEL MEDICINALE}

Rizmoic 200 microgrammi compresse rivestite con film.

\section{COMPOSIZIONE QUALITATIVA E QUANTITATIVA}

Ogni compressa contiene 200 microgrammi di naldemedina (come tosilato). Per l'elenco completo degli eccipienti, vedere paragrafo 6.1.

\section{FORMA FARMACEUTICA}

Compressa rivestita con film (compressa). Compressa rotonda, del diametro di circa $6,5 \mathrm{~mm}$, di colore giallo, con "222" e il logo Shionogi impressi su un lato e "0.2" sull'altro. 4. INFORMAZIONI CLINICHE

4.1 Indicazioni terapeutiche Rizmoic è indicato per i trattamento della stipsi indotta da oppioidi lOpioid-Induced Constipation, $\mathrm{OICl}$ nei pazienti adulti che sono stati trattati in precedenza con un lassativo. 4.2 Posologia e modo di somministrazione Posologia La dose raccomandata di naldemedina è 200 microgrammi (una compressa) al giorno. Rizmoic può essere usato con o senza lassativo(i). Può essere assunto in qualunque momento della giornata, ma s raccomanda di assumerlo ogni giorno alla stessa ora. Non è richiesta una modifica del regime posologico degli analgesici prima di iniziare il trattamento con Rizmoic. L'assunzione di Rizmoic deve essere interrotta se si interrompe il trattamento con l'analgesico oppioide. Popolazioni speciali Pazienti anziani Non è richiesto un aggiustamento della dose nei pazienti di età superiore a 65 anni (vedere paragrafo 5.2). A causa della limitata esperienza terapeutica nei pazienti di età pari o superiore a 75 anni, la terapia con naldemedina deve essere iniziata con cautela in questa fascia d'età. Compromissione renale Non è richiesto un aggiustamento della dose nei pazienti con compromissione renale (vedere paragrafo 5.2). A causa della limitata esperienza terapeutica, i pazienti con compromissione renale grave devono essere sottoposti a monitoraggio clinico quando iniziano la terapia con naldemedina. Compromissione epatica Non è richiesto un aggiustamento della dose nei pazienti con insufficienza epatica lieve o moderata. L'uso nei pazienti con compromissione epatica grave non è raccomandato (vedere paragrafi 4.4 e 5.2). Analgesicioppioidi L'esperienza in pazienti trattati con uno o più analgesic oppioidi a dosi giornaliere superiori a $400 \mathrm{mg}$ equivalenti di morfina è limitata. Non vi è esperienza in pazienti trattati per stipsi indotta da agonisti parziali dei recettori mu-oppioid lad es. buprenorfinal. Popolazione pediatrica La sicurezza e l'efficacia di naldemedina nei bambini e negli adolescenti di età inferiore a 18 anni non sono state ancora stabilite. Non ci sono dati disponibili. Modo di somministrazione Uso orale. Rizmoic deve essere assunto una volta al giorno, con o senza cibo (vedere paragrafo 5.2). 4.3 Controindicazioni Ipersensibilità al principio attivo o ad uno qualsiasi degli eccipienti elencati al paragrafo 6.1. Pazienti con accertata o sospetta ostruzione o perforazione gastrointestinale o pazienti a più elevato rischio di ostruzione ricorrente, a causa del potenziale rischio di perforazione gastrointestinale (vedere paragrafo 4.4). 4.4 Avvertenze speciali e precauzioni d'impiego Perforazione gastrointestinale Casi di perforazione gastrointestinale, inclusi casi con esito fatale, sono stati segnalati nel contesto post-marketing quando naldemedina è stata utilizzata in pazienti a più elevato rischio di perforazione gastrointestinale (GI) lad es. malattia diverticolare e sottostanti tumori maligni del tratto gastrointestinale o metastasi peritoneali). Naldemedina non deve essere utilizzata in pazienti con accertata o sospetta ostruzione GI 0 in pazienti a più elevato rischio di ostruzione ricorrente, a causa del potenziale rischio di perforazione GI lvedere paragrafo 4.3). L'uso di naldemedina richiede cautela nei pazienti affetti da qualsiasi patologia che può determinare una compromissione dell'integrità della parete del tratto gastrointestinale lad es. malattia peptica ulcerosa, sindrome di Ogilvie, tumori maligni del tratto GI, morbo di Crohn). Si deve tenere conto del rapporto beneficio/rischio complessivo per ciascun paziente. I pazienti devono essere monitorati per lo sviluppo di dolore addominale severo, persistente o in peggioramento. Qualora si sospetti ostruzione o perforazione, il trattamento con naldemedina deve essere interrotto (vedere paragrafo 4.3). Reazioni avverse gastrointestinali Reazioni avverse addominali lad es. dolore addominale, vomito e diarreal sono state segnalate con Rizmoic. I pazienti devono essere avvisati di riferire al proprio medico sintomi severi, persistenti o in peggioramento. In caso di diarrea o dolore addominale di grado severo, il paziente deve essere monitorato e trattato per la disidratazione, mediante reidratazione e terapia appropriata se necessario (vedere paragrafo 4.8). Sindrome di astinenza da oppioidi La sindrome di astinenza da oppioidi è un gruppo di tre o più dei segni o sintomi seguenti: umore disforico, nausea o vomito, dolori muscolari, lacrimazione o rinorrea, dilatazione pupillare o piloerezione o sudorazione, diarrea, sbadigli, febbre o insonnia. La sindrome di astinenza da oppioidi si sviluppa in genere da alcuni minuti a diversi giorni dopo la somministrazione di un antagonista degli oppioidi. Occorre usare cautela riguardo alla sospensione degli oppioidi. I pazienti devono essere avvisati di interrompere il trattamento con naldemedina e contattare il medico se si verifica astinenza da oppioidi. Casi di possibile sindrome di astinenza da oppioidi sono stati segnalati nel programma clinico di naldemedina (vedere paragrafo 4.8). I pazienti con alterazioni della barriera ematoencefalica lad es. tumori maligni primitivi cerebrali, metastasi o altre condizioni infiammatorie a carico del sistema nervoso centrale (SNC), sclerosi multipla in fase attiva e malattia di Alzheimer in fase avanzatal possono presentare un rischio maggiore di astinenza da oppioidi o di ridotta analgesia. In questi pazienti, deve essere considerato il rischio-beneficio complessivo di naldemedina, con un attento monitoraggio dei sintomi di astinenza da oppioidi. Pazienti con patologie cardiovascolari Naldemedina non è stata studiata nel programma di studi clinici in pazienti con anamnesi recente di infarto miocardico, ictus o attacco ischemico transitorio nei 3 mesi precedenti lo screening. Questi pazienti devono essere sottoposti a monitoraggio clinico durante il trattamento con Rizmoic. Uno studio del QTc eseguito con naldemedina in volontari 
sani non ha indicato un prolungamento dell'intervallo QT. pazienti con fattori di rischio per patologie cardiovascolari non sono stati esclusi dal programma di studi clinici di naldemedina, con IMC $\geqslant 30 \mathrm{~kg} / \mathrm{m}^{2}$ e anamnesi positiva per ipertensione e/o dislipidemia che costituivano i fattori di rischio più comunemente segnalati. Compromissione epatica grave Naldemedina non è stata studiata nei pazienti con grave compromissione epatica. L'uso di naldemedina non è raccomandato in questi pazienti (vedere paragrafo 4.2). Uso concomitante con inibitori e induttori forti del CYP3A L'uso concomitante di naldemedina con inibitori forti del CYP3A lad es. succo di pompelmo, itraconazolo, ketoconazolo, ritonavir, indinavir, saquinavir, telitromicina e claritromicina) comporta un aumento dell'esposizione a naldemedina e può aumentare il rischio di reazioni avverse. L'uso concomitante con inibitori forti del CYP3A deve essere evitato. L'uso concomitante di naldemedina con induttori forti del CYP3A lad es. iperico (Hypericum perforatum), rifampicina, carbamazepina, fenobarbital e fenitoinal provoca una diminuzione dell'esposizione a naldemedina, con possibile riduzione della sua efficacia. L'uso concomitante con induttori forti del CYP3A non è raccomandato (vedere paragrafo 4.5). L'uso concomitante di naldemedina con induttori moderati del CYP3A lad es. efavirenz) non è stato accertato e richiede cautela (vedere paragrafo 4.5). Sodio Questo medicinale contiene meno di $1 \mathrm{mmol}$ (23 mg) di sodio per compressa, cioè essenzialmente 'senza sodio'. 4.5 Interazioni con altri medicinali ed altre forme d'interazione Effetti di altri medicinali su naldemedina Naldemedina è metabolizzata principalmente dal CYP3A, con il contributo parziale di UGT1A3, ed è un substrato della P-glicoproteina (P-gp) (vedere paragrafo 5.2). Interazioni con inibitori del CYP3A Itraconazolo, un inibitore forte del CYP3A, ha aumentato l'esposizione a naldemedina di 2,9 volte, con conseguente potenziale aumento del rischio di reazioni avverse. L'uso concomitante di inibitori forti del CYP3A, quali succo di pompelmo, itraconazolo, ketoconazolo, ritonavir indinavir, saquinavir, telitromicina e claritromicina, deve essere evitato. Qualora l'uso con inibitori forti del CYP3A sia inevitabile, monitorare le reazioni avverse (vedere paragrafo 4.4). L'uso concomitante di inibitori moderati del CYP3A, quali fluconazolo, può aumentare la concentrazione plasmatica di naldemedina. In caso di utilizzo con moderati inibitori del CYP3A, monitorare le reazioni avverse. Non esiste alcun rischio di interazione con l'uso concomitante di inibitori lievi del CYP3A. Interazione con induttori forti e moderati del CYP3A Rifampicina, un induttore forte del CYP3A, ha diminuito significativamente l'esposizione a naldemedina (dell'83\%). L'uso concomitante di induttori forti del CYP3A, quali iperico (Hypericum perforatum), rifampicina, carbamazepina, fenobarbital e fenitoina, non è raccomandato. L'uso concomitante di naldemedina con induttori moderati (ad es. efavirenz) non è stato accertato e i pazienti devono essere monitorati (vedere paragrafo 4.4). Interazione con inibitori forti della P-gp L'uso concomitante di inibitori della P-gp, quali ciclosporina, può aumentare le concentrazioni plasmatiche di naldemedina. Se naldemedina viene utilizzata con inibitori forti della P-gp, monitorare le reazioni avverse. 4.6 Fertilità, gravidanza e allattamento Gravidanza I dati relativi all'uso di naldemedina in donne in gravidanza non esistono. Gli studi sugli animali non indicano effetti dannosi diretti o indiretti di tossicità riproduttiva (vedere paragrafo 5.3). L'uso di naldemedina durante la gravidanza può accelerare l'astinenza da oppioidi nel feto, a causa dell'immaturità della barriera ematoencefalica fetale. Naldemedina non deve essere usata durante la gravidanza a meno che le condizioni cliniche della donna rendano necessario il trattamento con naldemedina. Allattamento Non è noto se naldemedina/ metaboliti siano escreti nel latte materno. I dati disponibili nei ratti hanno mostrato l'escrezione di naldemedina nel latte (vedere paragrafo 5.3). A dosi terapeutiche, la maggior parte degli oppioidi (ad es. morfina, meperidina, metadone) viene escreta nel latte materno in quantità minime. Esiste la possibilità teorica che naldemedina provochi astinenza da oppioidi in un neonato allattato con latte materno, la cui madre sta assumendo un agonista dei recettori oppioidi. Il rischio per il lattante non può essere escluso. Naldemedina non deve essere usata durante l'allattamento. Fertilità Non sono disponibili dati nell'uomo relativi all'effetto di naldemedina sulla fertilità. Naldemedina non ha dimostrato effetti avversi clinicamente rilevanti sulla fertilità o sulle prestazioni riproduttive in maschi e femmine di ratto (vedere paragrafo 5.3). 4.7 Effetti sulla capacità di guidare veicoli e sull'uso di macchinari Naldemedina non altera o altera in modo trascurabile la capacità di guidare veicoli e di usare macchinari. 4.8 Effetti indesiderati Riassunto del profilo di sicurezza Le reazioni avverse più comunemente segnalate nei pazienti con dolore cronico non oncologico e OIC sono state dolore addominale $(7,8 \%)$, diarrea $(5,9 \%)$, nausea $(3,6 \%)$ e vomito $(1,1 \%)$. La maggior parte di queste reazioni avverse gastrointestinali è stata di gravità da lieve a moderata e si è risolta senza l'interruzione del trattamento con naldemedina. Un caso grave di dolore addominale e un caso grave di nausea sono stati segnalati in pazienti con dolore cronico non oncologico e OIC. Le reazioni avverse più comunemente segnalate nei pazienti oncologici con OIC sono state diarrea $(24,5 \%)$ e dolore addominale $(3,9 \%)$. La maggior parte di queste reazioni avverse gastrointestinali è stata di gravità da lieve a moderata e si è risolta con il trattamento. Due casi gravi di diarrea sono stati segnalati in pazienti oncologici con OIC. Tabella delle reazioni avverse Le reazioni avverse con naldemedina 200 microgrammi compresse, in pazienti con dolore cronico non oncologico e OIC e in pazienti oncologici con OIC, segnalate negli studi clinici, sono presentate nelle tabelle in base alla classificazione per sistemi e organi secondo MedDRA. Le categorie di frequenza sono definite con la seguente convenzione: molto comune $(\geqslant 1 / 10)$, comune $(\geqslant 1 / 100$, $<1 / 10)$, non comune $(\geqslant 1 / 1.000,<1 / 100)$, raro $(\geqslant 1 / 10.000$, $<1 / 1.000$ ), molto raro $(<1 / 10.000$ ) e non nota (la frequenza non può essere definita sulla base dei dati disponibili). All'interno di ogni gruppo di frequenza, le reazioni avverse sono presentate in ordine di gravità decrescente.

Tabella 1. Reazioni avverse presentate secondo la classificazione per sistemi e organi e la frequenza in pazienti con dolore cronico non oncologico e stipsi indotta da oppioidi

\begin{tabular}{|l|l|l|l|l|}
\hline $\begin{array}{l}\text { Classificazione } \\
\text { per sistemi e } \\
\text { organi }\end{array}$ & Comune & $\begin{array}{l}\text { Non } \\
\text { comune }\end{array}$ & Raro & Non nota \\
\hline $\begin{array}{l}\text { Disturbi del sistema } \\
\text { immunitario }\end{array}$ & & Ipersensibilitàa & \\
\hline $\begin{array}{l}\text { Patologie } \\
\text { gastrointestinali }\end{array}$ & $\begin{array}{l}\text { Diarrea } \\
\text { Dolore } \\
\text { addominale }\end{array}$ & $\begin{array}{l}\text { Nausea } \\
\text { Vomito }\end{array}$ & $\begin{array}{l}\text { Perforazione } \\
\text { gastrointestinale }\end{array}$ \\
\hline $\begin{array}{l}\text { Patologie sistemiche } \\
\text { e condizioni } \\
\text { relative alla sede di } \\
\text { somministrazione }\end{array}$ & $\begin{array}{l}\text { Sindrome } \\
\text { di astinenza } \\
\text { da oppioidi }\end{array}$ & & \\
\hline
\end{tabular}


anna segnalazione grave di reazione di ipersensibilità è stata osservata negli studi clinici con naldemedina. Il paziente si è ripreso

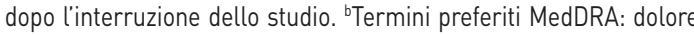
addominale, dolore addominale superiore, dolore addominale inferiore e fastidio addominale.

Tabella 2. Reazioni avverse presentate secondo la classificazione per sistemi e organi e la frequenza in pazienti oncologici con stipsi indotta da oppioidi

\begin{tabular}{|l|l|l|l|l|}
\hline $\begin{array}{l}\text { Classificazione } \\
\text { per sistemi e } \\
\text { organi }\end{array}$ & $\begin{array}{l}\text { Molto } \\
\text { comune }\end{array}$ & Comune & Non comune & Non nota \\
\hline $\begin{array}{l}\text { Patologie } \\
\text { gastrointestinali }\end{array}$ & Diarrea & $\begin{array}{l}\text { Dolore } \\
\text { addominale }\end{array}$ & & $\begin{array}{l}\text { Perforazione } \\
\text { gastrointestinale }\end{array}$ \\
\hline $\begin{array}{l}\text { Patologie sistemiche } \\
\text { e condizioni } \\
\text { relative alla sede di } \\
\text { somministrazione }\end{array}$ & & $\begin{array}{l}\text { Sindrome } \\
\text { di astinenza } \\
\text { da oppioidi }\end{array}$ & \\
\hline
\end{tabular}

aTermini preferiti MedDRA: dolore addominale, dolore addominale superiore, dolore addominale inferiore e fastidio addominale.

Descrizione di reazioni avverse selezionate Sindrome di astinenza da oppioidi Possibile astinenza da oppioidi, definita come almeno tre reazioni avverse potenzialmente correlate ad astinenza da oppioidi verificatesi lo stesso giorno e non correlate unicamente all'apparato gastrointestinale, si è manifestata nello $0,8 \%$ (9/1.163) dei pazienti con dolore cronico non oncologico e OIC che assumevano naldemedina, rispetto allo $0,2 \%(2 / 1.165)$ dei pazienti che assumevano placebo, indipendentemente dal trattamento di mantenimento con oppioidi, e nello $0,6 \%(1 / 155)$ dei pazienti oncologici con OIC che assumevano naldemedina 200 microgrammi, rispetto allo $0 \%(0 / 152)$ dei pazienti che assumevano placebo. I sintomi comprendevano, ma non erano limitati a, iperidrosi, brividi, lacrimazione aumentata, vampata di calore/rossore, piressia, starnuto, sensazione di freddo, dolore addominale, diarrea, nausea, vomito, artralgia, mialgia e tachicardia (vedere paragrafo 4.4). Patologie gastrointestinali Dolore addominale, diarrea, nausea e vomito sono state le reazioni avverse più comunemente segnalate negli studi clinici condotti in pazienti con dolore cronico non oncologico e OIC e in pazienti oncologici con OIC. La maggior parte di queste reazioni avverse gastrointestinali è stata di severità lieve o moderata e si è risolta con il trattamento. Il tasso di interruzione dovuto ad eventi avversi gastrointestinali emergenti dal trattamento con naldemedina 200 microgrammi, rispetto al placebo, è stato rispettivamente del $3,2 \%$ e $1 \%$ nei pazienti con dolore cronico non oncologico e OIC, e rispettivamente del $4,5 \%$ e $0 \%$ per i pazienti oncologici con OIC. Segnalazione delle reazioni avverse sospette La segnalazione delle reazioni avverse sospette che si verificano dopo l'autorizzazione del medicinale è importante, in quanto permette un monitoraggio continuo del rapporto beneficio/rischio del medicinale. Agli operatori sanitari è richiesto di segnalare qualsiasi reazione avversa sospetta tramite l'Agenzia Italiana del Farmaco. Sito web: https://www.aifa.gov.it/content/segnalazioni-reazioniavverse 4.9 Sovradosaggio Volontari sani Una dose singola di naldemedina fino a $100 \mathrm{mg}$ e dosi ripetute fino a $30 \mathrm{mg} /$ die per 10 giorni sono state somministrate a volontari sani negli studi clinici. Sono stati osservati aumenti dose- dipendenti delle reazioni avverse di natura gastrointestinale inclusi dolore addominale, diarrea e nausea. Le reazioni sono state di gravità lieve o moderata e si sono risolte. Pazienti con OIC Una dose singola di naldemedina (da $0,01 \mathrm{mg}$ a $3 \mathrm{mgl}$ e dosi ripetute di $0,4 \mathrm{mg} /$ die sono state somministrate a pazienti con OIC negli studi clinici. Un paziente che ha assunto una dose singola di naldemedina da $1 \mathrm{mg}$ ha manifestato grave sindrome di astinenza da farmaco, comprendente nausea e crampi allo stomaco, ed è stato trattato con esomeprazolo e ondansetron per la nausea e midazolam cloridrato per i crampi allo stomaco. I sintomi si sono risolti. Negli studi clinici, i pazienti con OIC trattati con $0,4 \mathrm{mg} /$ die (il doppio della dose raccomandata) nell'arco di 4 settimane hanno presentato una maggiore incidenza di reazioni avverse al farmaco di natura gastrointestinale, inclusi diarrea e dolore addominale, spesso entro 1-2 giorni dalla somministrazione iniziale. Gestione Non esiste un antidoto specifico per la naldemedina. Naldemedina non viene eliminata dall'organismo mediante emodialisi. In caso di sovradosaggio, i pazienti devono essere attentamente monitorati per rilevare potenziali segni e sintomi di sindrome di astinenza da oppioidi (vedere paragrafo 4.4) e ricevere terapie di supporto appropriate.

\section{PROPRIETÀ FARMACOLOGICHE}

5.1 Proprietà farmacodinamiche Categoria farmacoterapeutica: farmaci per la stipsi, antagonisti dei recettori oppioidi periferici, codice ATC: A06AHO5. Meccanismo d'azione Naldemedina è un'antagonista degli oppioidi che si lega ai recettori oppioidi mu, delta e kappa. Naldemedina funziona come antagonista dei recettori oppioidi mu ad azione periferica, in tessuti quali il tratto gastrointestinale, riducendo così gli effetti costipanti degli oppioidi senza invertirne gli effetti mediati dal sistema nervoso centrale (SNC). Naldemedina è un derivato di naltrexone, a cui è stata aggiunta una catena laterale che aumenta il peso molecolare e l'area di superficie polare, riducendone così la capacità di attraversare la barriera ematoencefalica (BEE); la penetrazione di naltrexone a livello del SNC si prevede trascurabile alla dose raccomandata. Inoltre, naldemedina è un substrato del trasportatore di efflusso P-glicoproteina (P-gp), che può anche essere coinvolto nel ridurre la penetrazione di naldemedina nel SNC. Sulla base di questi dati, si prevede che naldemedina eserciti i propri effetti anticostipanti sugli oppioidi, senza invertirne gli effetti analgesici mediati dall'SNC. Efficacia e sicurezza clinica L'efficacia e la sicurezza di naldemedina sono state stabilite in pazienti con dolore cronico non oncologico e $\mathrm{OIC}$ e in pazienti oncologici con OIC. Studi clinici in pazienti con dolore cronico non oncologico e OIC La sicurezza e l'efficacia di naldemedina sono state valutate in due studi identici della durata di 12 settimane randomizzati, in doppio cieco, controllati verso placebo (Studi V9231 e V9232), in cui naldemedina è stata utilizzata senza lassativi, e in un terzo studio a lungo termine della durata di 52 settimane randomizzato, in doppio cieco, controllato verso placebo (Studio V9235), in cui naldemedina è stata utilizzata con o senza lassativi stabili in pazienti con dolore cronico non oncologico e OIC. Erano eleggibili a partecipare pazienti trattati con oppioidi a una dose giornaliera stabile equivalente di morfina di $\geqslant 30 \mathrm{mg}$ per almeno 4 settimane prima dell'arruolamento e con OIC auto-riferita. Negli Studi V9231 e V9232, l'OIC è stata confermata in un periodo preliminare di 2 settimane ed era definita come non più 
di 4 movimenti intestinali spontanei (Spontaneous Bowel Movements, SBM) totali, nell'arco di 14 giorni consecutivi, e $<3$ SBM in una data settimana con almeno il 25\% degli SBM associati a una o più delle seguenti condizioni: (1) sforzo, (2) feci dure o grumose, (3) sensazione di evacuazione incompleta e (4) sensazione di ostruzione/blocco anorettale. Nello Studio V9235, l'OIC è stata confermata attraverso un periodo preliminare di 2 settimane ed era definita come non più di 4 SBM totali nell'arco di 14 giorni consecutivi, e <3 SBM in una data settimana. Un SBM era definito come un movimento intestinale senza assunzione di lassativo di salvataggio nelle 24 ore precedenti. Negli Studi V9231 e V9232, i pazienti dovevano in alternativa non essere in trattamento con lassativi oppure essere disposti a interrompere i lassativi al momento dello Screening e a utilizzare solo i lassativi di salvataggio forniti durante i periodi di Screening e di Trattamento. Tutti i partecipanti allo studio avevano assunto in precedenza lassativi per il trattamento dell'OIC. Nello Studio V9235, ai pazienti in regime stabile di lassativo allo screening $(52,4 \%)$ è stato consentito di continuare l'uso dello stesso regime senza modifiche per tutta la durata dello studio. Nei periodi preliminare e di trattamento per tutti i tre studi, è stato usato bisacodile come lassativo di salvataggio se i pazienti non avevano movimenti intestinali da 72 ore, con la possibilità di ricorrere una sola volta ad un clistere in assenza di movimenti intestinali dopo 24 ore dall'assunzione di bisacodile. I pazienti con evidenza di anomalie strutturali significative del tratto gastrointestinale non sono stati arruolati in questi studi. In totale, 547 pazienti nello Studio V9231, 551 pazienti nello Studio V9232 e 1.246 pazienti nello Studio V9235 sono stati randomizzati in rapporto 1:1 a ricevere 200 microgrammi di naldemedina - placebo una volta al giorno per 12 settimane per gli Studi V9231 e V9232, e per 52 settimane per lo Studio V9235. Negli Studi V9231, V9232 e V9235, l'età media dei soggetti in questi tre studi era 53,2 anni; il 14,8\% aveva 65 anni di età o più, il $62,0 \%$ era costituito da donne e l' $80,2 \%$ era bianco. Nello Studio V9231, i tre tipi più comuni di dolore erano dolore dorsale $(62,0 \%)$, dolore al collo $(8,3 \%)$ e osteoartrite $(5,3 \%)$. Nello Studio V9232, erano dolore dorsale $(53,6 \%)$, dolore $(10,2 \%)$ e artralgia $(7,8 \%)$. Nello Studio V9235, i tre tipi più comuni di dolore erano dolore dorsale $(58,0 \%)$, osteoartrite $(9,5 \%)$ e dolore al collo $(8,1 \%)$. Prima dell'arruolamento, i pazienti utilizzavano il loro oppioide corrente in media da 5 anni. I pazienti che hanno partecipato agli Studi V9231, V9232 e V9235 assumevano una vasta gamma di oppioidi. La dose media equivalente di morfina giornaliera di oppioidi al basale era $132,42 \mathrm{mg}$, $120,93 \mathrm{mg}$ e $122,06 \mathrm{mg}$ al giorno rispettivamente per gli Studi V9231, V9232 e V9235. Gli SBM medi al basale erano 1,31, 1,17 e 1,60, rispettivamente per gli Studi V9231. V9232 e V9235. L'endpoint primario per gli Studi V9231 e V9232 era la proporzione di responder SBM, definita come: $\geqslant 3$ SBM alla settimana e una variazione rispetto al basale di $\geqslant 1$ SBM alla settimana, per almeno 9 delle 12 settimane di studio e 3 delle ultime 4 settimane. L'endpoint primario di efficacia per lo Studio V9235 era la variazione della frequenza settimanale di movimenti intestinali, dal basale alle Settimane 12, 24, 36 e 52. Vi è stata una differenza statisticamente significativa per il gruppo di trattamento con naldemedina, rispetto al placebo, per l'endpoint primario negli Studi V9231 e V9232 (vedere Tabella 3). Negli Studi V9231 e V9232 vi erano 4 endpoint secondari (vedere Tabella 3).
Tabella 3. Esiti clinici per gli studi V9231 e V9232

\begin{tabular}{|c|c|c|c|c|}
\hline & \multicolumn{2}{|c|}{ V9231 } & \multicolumn{2}{|c|}{ V9232 } \\
\hline & $\begin{array}{l}\text { Naldemedina } \\
\qquad(N=273)\end{array}$ & $\begin{array}{l}\text { Placebo } \\
(\mathrm{N}=272)\end{array}$ & $\begin{array}{c}\text { Naldemedina } \\
(N=276)\end{array}$ & $\begin{array}{l}\text { Placebo } \\
(\mathrm{N}=274)\end{array}$ \\
\hline $\begin{array}{l}\text { Proporzione di responder } \\
\text { SBM }\end{array}$ & $47,6 \%$ & $34,6 \%$ & $52,5 \%$ & $33,6 \%$ \\
\hline Differenza di trattamento & \multicolumn{2}{|c|}{$\begin{array}{c}13,0 \% \\
\text { (IC al } 95 \%: 4,8 \%, 21,3 \%, \\
\left.p=0,0020^{*}\right)\end{array}$} & \multicolumn{2}{|c|}{$\begin{array}{c}18,9 \% \\
\text { (IC al 95\%: } 10,8 \%, 27,0 \% \\
p<0,0001^{*} \text { ) }\end{array}$} \\
\hline \multicolumn{5}{|c|}{ Variazione della frequenza settimanale di SBM (media dei minimi quadrati) } \\
\hline $\begin{array}{l}\text { Dal basale alle ultime } \\
2 \text { settimane di trattamento** }\end{array}$ & 3,42 & 2,12 & 3,56 & 2,16 \\
\hline Dal basale alla settimana $1^{* *}$ & 3,48 & 1,36 & 3,86 & 1,69 \\
\hline \multicolumn{5}{|c|}{ Variazione della frequenza settimanale di CSBM (media dei minimi quadrati) } \\
\hline $\begin{array}{l}\text { Dal basale alle ultime } \\
2 \text { settimane di trattamento** }\end{array}$ & 2,58 & 1,57 & 2,77 & 1,62 \\
\hline \multicolumn{5}{|c|}{$\begin{array}{l}\text { Variazione della frequenza settimanale di SBM senza sforzo (media dei minimi } \\
\text { quadrati) }\end{array}$} \\
\hline $\begin{array}{l}\text { Dal basale alle ultime } \\
2 \text { settimane di trattamento*** }\end{array}$ & 1,46 & 0,73 & 1,85 & 1,10 \\
\hline
\end{tabular}

IC = intervallo di confidenza;

*Statisticamente significativo: valori basati sul test di Cochran-MantelHaenszel.

** $p<0,0001$. ${ }^{* * *} p=0,0003$ per lo studio V9231 e $p=0,0011$ per lo studio V9232.

Per lo Studio V9235, l'efficacia di naldemedina rispetto al placebo è stata valutata come endpoint secondario dalla frequenza di movimenti intestinali, come riportato nella Tabella 4.

Tabella 4. Variazione della frequenza settimanale di movimenti intestinali dal basale a ciascuna visita (media dei minimi quadrati) Popolazione ITT nello Studio V9235

\begin{tabular}{|l|c|c|}
\cline { 2 - 3 } \multicolumn{1}{l|}{} & $\begin{array}{c}\text { Naldemedina } \\
\mathbf{( N = 6 2 1 )}\end{array}$ & $\begin{array}{c}\text { Placebo } \\
\text { (N = 620) }\end{array}$ \\
\hline $\begin{array}{l}\text { Frequenza media di movimenti intestinali } \\
\text { al basale }\end{array}$ & 2,02 & 2,02 \\
\hline $\begin{array}{l}\text { Variazione della frequenza settimanale } \\
\text { di movimenti intestinali }\end{array}$ & & \\
\hline Settimana $12^{*}$ & 3,70 & 2,42 \\
\hline Settimana 24* & 3,77 & 2,77 \\
\hline Settimana 36* & 3,88 & 2,88 \\
\hline Settimana 52* & 3,92 & 2,92 \\
\hline
\end{tabular}

*p nominale $\leqslant 0,0001$.

L'efficacia e la sicurezza sono state valutate anche nei responder inadeguati ai lassativi (Laxative Inadequate Responders, LIR) e nei sottogruppi non-LIR. Negli Studi V9231 e V9232, venivano considerati LIR i pazienti che, sulla base dei medicinali concomitanti documentati, erano in terapia con lassativi prima dell'ingresso nello 
studio e che ne avevano interrotto l'uso entro i 30 giorni precedenti lo Screening, ed avevano OIC auto-riferita. Inoltre, erano considerati non-LIR i pazienti che non erano in terapia con lassativi nei 30 giorni precedenti lo Screening e che avevano ricevuto solo il lassativo di salvataggio allo Screening o dopo lo Screening. II numero di pazienti nei sottogruppi LIR e non-LIR era pari a 629 (naldemedina: 317 e placebo: 312) e a 451 (naldemedina: 223 e placebo: 228) per gli Studi V9231 e V9232 combinati. Tutti i partecipanti allo studio avevano assunto in qualche momento lassativi per il trattamento dell'OIC prima dell'ingresso negli studi V9231 o V9232. $\mathrm{Nel}$ sottogruppo LIR, è stata osservata una proporzione maggiore di responder con naldemedina $(46,4 \%)$ rispetto al placebo $(30,2 \%)$ e la differenza tra i gruppi $(16,2 \%)$ è stata statisticamente significativa ( $p<0,0001$ ). Nel sottogruppo non-LIR, coerentemente con i risultati nel sottogruppo LIR, è stata osservata una proporzione maggiore di responder con naldemedina $(54,3 \%)$ rispetto al placebo $(38,9 \%)$ e la differenza tra i gruppi $(15,6 \%)$ è stata statisticamente significativa $(p=0,0009)$. Per lo Studio V9235, i dati di efficacia a lungo termine, definita come variazione della frequenza di movimenti intestinali alla settimana 52 rispetto al basale, valutata come endpoint secondario, hanno evidenziato miglioramenti della frequenza di movimenti intestinali nei soggetti del gruppo trattato con naldemedina, rispetto ai soggetti del gruppo placebo, nei sottogruppi LIR $(3,10$ vs $1,90, p=0,0210)$ e non-LIR $(4,26$ vs $3,39, p=0,1349)$. Studi clinici in pazienti oncologici con OIC La sicurezza e l'efficacia di naldemedina sono state valutate inoltre in 2 studi randomizzati, in doppio cieco e controllati verso placebo (V9222 e V9236), in pazienti oncologici con OIC. I soggetti dovevano essere in trattamento con oppioidi da $\geqslant 14$ giorni prima dello Screening e dovevano ricevere una dose stabile. Gli studi prevedevano un Periodo di screening di 2 settimane, un Periodo di trattamento di 2 settimane e un Periodo di follow-up di 4 settimane. Per i pazienti in terapia con lassativi alla visita di Screening, la terapia doveva essere continuata a una dose stabile fino al termine del Periodo $d$ trattamento. Ai pazienti era consentito ricevere uno o più lassativi di salvataggio, al bisogno, indipendentemente dal fatto che essi seguissero un regime con lassativi stabile al basale leccetto entro 24 ore dall'inizio del Periodo di trattamento). Negli studi V9222 e V9236, l'OIC è stata confermata in un periodo preliminare di 2 settimane ed era definita come $\leqslant 5$ SBM, nell'arco di 14 giorni consecutivi prima della randomizzazione, $e \geqslant 1$ dei seguent sintomi intestinali in $\geqslant 25 \%$ di tutti i movimenti intestinali, indipendentemente dall'uso di lassativi di salvataggio: presenza di sforzo durante il movimento intestinale sensazione di evacuazione incompleta, evacuazione $d i$ feci dure o piccoli granuli. Negli Studi V9222 e V9236, l'età media dei soggetti era 64,3 anni; il $51,8 \%$ aveva 65 anni di età o più, il $39,4 \%$ era costituito da donne e il $97,1 \%$ era giapponese. Naldemedina 200 microgrammi o placebo sono stati somministrati per 2 settimane ai pazienti oncologici con OIC. L'endpoint primario per lo Studio V9236 e l'endpoint secondario, senza aggiustamento per la molteplicità, per lo Studio V9222 erano la proporzione di responder SBM durante il Periodo di trattamento di 2 settimane. Un responder era definito come un paziente con una frequenza settimanale di SBM $\geqslant 3$ e un aumento rispetto al basale di SBM settimanal $\geqslant 1$, durante il Periodo di trattamento di 2 settimane.
Tabella 5. Proporzione di responder SBM nei pazienti oncologici con OIC durante il periodo di trattamento di 2 settimane (Studi V9222 e V9236)

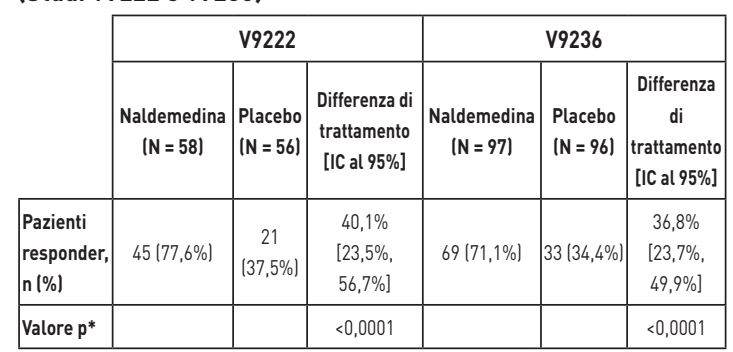

*Statisticamente significativo: valori basati sul test Chi-quadrato.

Popolazione pediatrica L'Agenzia europea dei medicinali ha rinviato l'obbligo di presentare i risultati degli studi con Rizmoic in uno o più sottogruppi della popolazione pediatrica per il trattamento della stipsi indotta da oppioidi (vedere paragrafo 4.2 per informazioni sull'uso pediatrico). $\mathbf{5 . 2}$ Proprietà farmacocinetiche Assorbimento Naldemedina è assorbita con un tempo al raggiungimento del picco di concentrazione plasmatica pari a circa 0,75 ore a digiuno. La biodisponibilità assoluta di naldemedina non è stata stabilita. Si stima che la biodisponibilità assoluta di naldemedina sia compresa in un intervallo dal $20 \%$ al $56 \%$. Il cibo non ha alcun effetto clinicamente significativo. Il picco di concentrazione plasmatica si è ridotto del $35 \%$ e il tempo al raggiungimento del picco di concentrazione plasmatica è stato ritardato da 0,75 ore a digiuno a 2,5 ore a stomaco pieno, mentre nessuna differenza significativa è stata osservata nell'area sotto la curva concentrazione plasmatica/tempo con l'assunzione di cibo. Sulla base di questi dati, naldemedina può essere assunta con o senza cibo (vedere paragrafo 4.2). Distribuzione Naldemedina è altamente legata alle proteine sieriche, prevalentemente all'albumina sierica umana e, in misura minore, alla glicoproteina $\alpha-1$ acida e alla $\gamma$-globulina, con un rapporto medio di legame alle proteine nell'uomo del 93,2\%. Il volume di distribuzione apparente è circa 155 litri. Biotrasformazione Naldemedina è metabolizzata principalmente dal CYP3A in nor-naldemedina, con un contributo di minore entità dell'UGT1A3 a formare naldemedina 3-G. Dopo la somministrazione orale di naldemedina marcata con [14C], il metabolita primario nel plasma è stata la nor-naldemedina, con un'esposizione relativa rispetto a naldemedina compresa tra circa il 9 e il $13 \%$. Naldemedina 3-G era un metabolita minore nel plasma, con un'esposizione relativa rispetto a naldemedina inferiore al $3 \%$. Naldemedina subisce inoltre una scissione nel tratto gastrointestinale, per formare benzamidina e acido carbossilico di naldemedina. Negli studi in vitro a concentrazioni clinicamente rilevanti, naldemedina non ha inibito i principali enzimi del CYP (inclusi gli isoenzimi CYP1A2, CYP2A6, CYP2B6, CYP2C8, CYP2C9, CYP2C19, CYP2D6, CYP2E1, CYP3A o CYP4A11) e non è un inibitore dei trasportatori OATP1B1, OATP1B3, 0AT1, OAT3, OCT1, OCT2, BCRP, P-gp, MATE1, MATE2-K o BSEP. Naldemedina non ha causato un' induzione significativa degli isoenzimi CYP1A2, CYP2B6 o CYP3A4. Pertanto, non si prevede che il trattamento con naldemedina alteri la farmacocinetica dei medicinali somministrati in concomitanza che sono substrati di questi enzimi e trasportatori. Eliminazione L'emivita di eliminazione terminale apparente di naldemedina è di circa 11 ore, mentre la clearance totale apparente (CL/F) di naldemedina è pari a $8,4 \mathrm{~L} / \mathrm{h}$. Dopo la somministrazione 
orale di naldemedina radiomarcata, rispettivamente il $57,3 \%$ e il $34,8 \%$ della dose sono stati escreti nelle urine e nelle feci per [oxadiazolo- ${ }^{14} \mathrm{C}$ ]-naldemedina e il $20,4 \%$ e il $64,3 \%$ della dose sono stati escreti, rispettivamente, come [carbonil-14 $\mathrm{C}$ ]naldemedina nelle urine e nelle feci. Circa il $20 \%$ della dose di naldemedina viene escreto immodificato nelle urine. Linearità/ Non linearità Il picco di concentrazione plasmatica e l'area sotto la curva concentrazione plasmatica-tempo sono aumentati in modo quasi proporzionale alla dose nell'intervallo di dose da 0,1 a $100 \mathrm{mg}$. Un leggero accumulo (da 1 a 1,3 volte) per il picco di concentrazione plasmatica e l'area sotto la curva concentrazione plasmatica-tempo è stato osservato dopo la somministrazione di dosi multiple una volta al giorno, a digiuno, per 10 giorni. Farmacocinetica in sottopopolazioni Età, sesso, peso corporeo ed etnia Un'analisi di farmacocinetica di popolazione derivata dagli studi clinici con naldemedina non ha individuato un effetto clinicamente significativo di età, sesso, peso corporeo o etnia sulla farmacocinetica di naldemedina. La farmacocinetica di naldemedina nella popolazione pediatrica non è stata studiata (vedere paragrafo 4.2). Compromissione renale La farmacocinetica di naldemedina dopo la somministrazione di una dose singola di 200 microgrammi di naldemedina è stata studiata in soggetti con compromissione renale lieve, moderata o grave, o con malattia renale allo stadio terminale (End-Stage Renal Disease, ESRD) che necessitavano di emodialisi, e confrontata con soggetti sani con funzione renale nella norma. La farmacocinetica di naldemedina è risultata simile tra i soggetti con compromissione renale lieve, moderata o grave, o i soggetti con ESRD che necessitavano di emodialisi, e i soggetti sani con funzione renale nella norma. Le concentrazioni plasmatiche di naldemedina in soggetti con ESRD che necessitavano di dialisi sono risultate simili quando naldemedina è stata somministrata prima o dopo l'emodialisi, indicando che naldemedina non viene eliminata dall'organismo con questa procedura. Compromissione epatica L'effetto della compromissione epatica sulla farmacocinetica di una dose singola di 200 microgrammi di naldemedina è stato studiato in soggetti con compromissione epatica classificata come lieve (Child-Pugh classe A) o moderata (Child-Pugh classe B) e confrontato con soggetti sani con funzione epatica nella norma. La farmacocinetica di naldemedina è risultata simile tra i soggetti con compromissione epatica lieve o moderata e i soggetti sani con funzione epatica nella norma. L'effetto della compromissione epatica grave (ChildPugh classe C) sulla farmacocinetica di naldemedina non è stato valutato. 5.3 Dati preclinici di sicurezza I dati preclinici non rivelano rischi particolari per l'uomo sulla base di studi convenzionali di sicurezza farmacologica, tossicità a dosi ripetute, genotossicità, potenziale cancerogeno e sviluppo embrio-fetale. Nello studio sulla fertilità e il primo sviluppo embrionale nel ratto, un prolungamento della fase diestrale è stato osservato a $10 \mathrm{mg} / \mathrm{kg} / \mathrm{die}$, ma non è stato osservato a $1 \mathrm{mg} / \mathrm{kg} / \mathrm{die}$ (12 volte l'esposizione [AUC ${ }_{0-24 \mathrm{hr}}$ ] nell'uomo a una dose orale di 200 microgrammi). L'effetto sul ciclo estrale non è considerato clinicamente pertinente alla dose terapeutica proposta. Non sono stati osservati effetti avversi sulla fertilità e sulle prestazioni riproduttive maschili o femminili a una dose fino a $1.000 \mathrm{mg} / \mathrm{kg} /$ die loltre 16.000 volte l'esposizione [AUC ${ }_{0-24 h r}$ ] nell'uomo a una dose orale di 200 microgrammi). Nello studio sullo sviluppo pre- e postnatale nel ratto, una madre è morta al momento del parto a una dose di
$1.000 \mathrm{mg} / \mathrm{kg} / \mathrm{die}$, mentre scarso allattamento, soppressione dell'incremento del peso corporeo e riduzione del consumo di cibo sono stati osservati a 30 e $1.000 \mathrm{mg} / \mathrm{kg} / \mathrm{die}$. Riduzioni dell'indice di vitalità al Giorno 4 dopo la nascita sono state osservate a 30 e $1.000 \mathrm{mg} / \mathrm{kg} / \mathrm{die}$, mentre basso peso corporeo e ritardato dispiegamento del padiglione auricolare sono stati osservati a $1.000 \mathrm{mg} / \mathrm{kg} / \mathrm{die}$ nei cuccioli. Non vi sono stati effetti avversi sullo sviluppo pre- e postnatale a $1 \mathrm{mg} /$ $\mathrm{kg} /$ die (12 volte l'esposizione [AUC ${ }_{0-24 \mathrm{hr}}$ ] nell'uomo a una dose orale di 200 microgrammil. Il passaggio transplacentare di radioattività derivata da [carbonil-14 $\mathrm{C}$ ]-naldemedina è stato osservato in femmine di ratto gravide. La radioattività derivata da [carbonil- ${ }^{14} \mathrm{C}$ ]-naldemedina è stata escreta nel latte nei ratti in allattamento. Negli studi di tossicità giovanile condotti nei ratti, agli stessi livelli di dose, l'esposizione negli animali giovani ( $10^{\circ}$ giorno post-natale) è risultata aumentata rispetto agli animali adulti (da 2,3 a 7,4 volte). Nuovi rilievi istopatologici sono stati osservati a tutte le dosi testate nelle femmine di ratto, nelle ovaie (follicoli terziari/cisti luteiniche), oltre a cicli estrali irregolari, iperplasia della ghiandola mammaria e mucificazione vaginale già osservati negli animali adulti (la dose minima testata corrispondeva a un margine di esposizione di 6 o superiore, a seconda dell'età dei cuccioli). È stata osservata inoltre apertura vaginale anticipata di tre giorni, indicativa di un esordio precoce della maturità sessuale, ma solo a esposizioni elevate ritenute sufficientemente superiori all'esposizione umana massima, a una dose orale di 200 microgrammi.

\section{INFORMAZIONI FARMACEUTICHE}

6.1 Elenco degli eccipienti Nucleo della compressa Mannitolo, Croscarmellosa sodica, Magnesio stearato. Film di rivestimento Ipromellosa, Talco, Ossido di ferro giallo (E172). 6.2 Incompatibilità Non pertinente. $\mathbf{6 . 3}$ Periodo di validità 3 anni. 6.4 Precauzioni particolari per la conservazione Questo medicinale non richiede alcuna temperatura particolare di conservazione. Conservare nella confezione originale per proteggere il medicinale dalla luce e dall'umidità. 6.5 Natura e contenuto del contenitore Blister in alluminio/alluminio contenente 7, 10 o 14 compresse rivestite con film. Confezioni

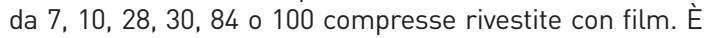
possibile che non tutte le confezioni siano commercializzate. 6.6 Precauzioni particolari per lo smaltimento Nessuna istruzione particolare.

\section{TITOLARE DELL'AUTORIZZAZIONE ALL'IMMISSIONE IN COMMERCIO}

Shionogi B.V.

Kingsfordweg 151

1043GR Amsterdam

Paesi Bassi

\section{NUMERO(I) DELL'AUTORIZZAZIONE ALL'IMMISSIONE IN COMMERCIO}

EU/1/18/1291/001, EU/1/18/1291/002, EU/1/18/1291/003, EU/1/18/1291/004, EU/1/18/1291/005,EU/1/18/1291/006.

9. DATA DELLA PRIMA AUTORIZZAZIONE/RINNOVO

\section{DELL'AUTORIZZAZIONE}

Data della prima autorizzazione: 18 febbraio 2019.

\section{DATA DI REVISIONE DEL TESTO}

30 Gennaio 2020.

Informazioni più dettagliate su questo medicinale sono disponibili sul sito web dell'Agenzia europea

dei medicinali, http://www.ema.europa.eu.

\begin{tabular}{|l|l|l|l|l|}
\hline Confezione & A.I.C. & Classe & Regime di fornitura & Prezzo al pubblico \\
\hline $\begin{array}{l}\text { Rizmoic } 200 \text { microgrammi compresse } \\
\text { rivestite con film - 28 compresse }\end{array}$ & $047626027 / \mathrm{E}$ & A - Nota AIFA 90 & RR & $€ 93,81^{*}$ \\
\hline
\end{tabular}

${ }^{*}$ Al lordo delle riduzioni previste per legge 


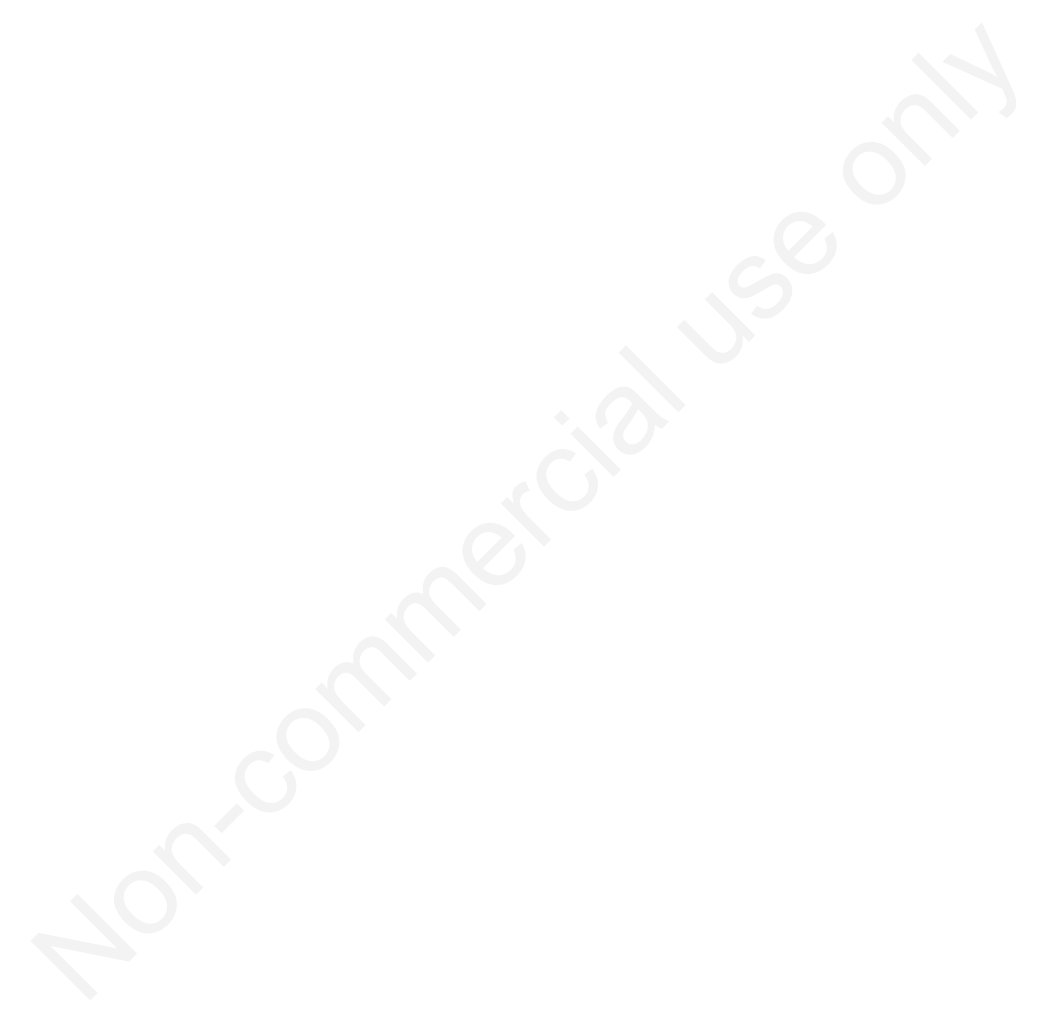




\section{粉 Rizmoic $^{\text {v }}$ \\ (naldemedina)}

\section{UNA COMPRESSA AL GIORNO PER IL TRATTAMENTO DELLA STIPSI INDOTTA DA OPPIOIDI (OIC) NEI PAZIENTI ADULTI CHE SONO STATI TRATTATI IN PRECEDENZA CON UN LASSATIVO'}

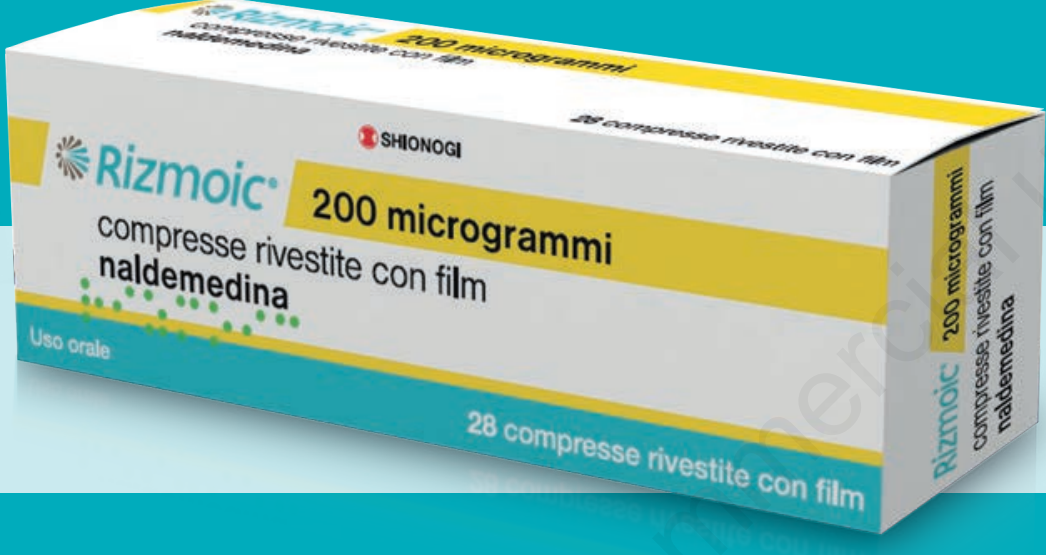

\section{Classe: A - Nota AIFA 90}

Regime di dispensazione: medicinale soggetto a prescrizione medica (RR).

Prezzo: 93,81€*

*Al lordo delle riduzioni previste per legge.

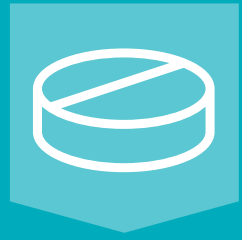

Una compressa al giorno $(200 \mu \mathrm{g}) .^{1}$

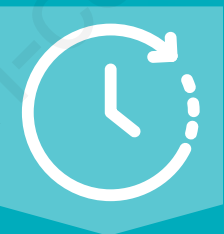

RIZMOIC ${ }^{\circledR}$ può essere assunto IN QUALUNQUE MOMENTO DELLA GIORNATA."

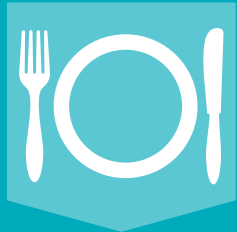

RIZMOIC ${ }^{\circledR}$ può essere assunto CON O SENZA CIBO. ${ }^{1}$

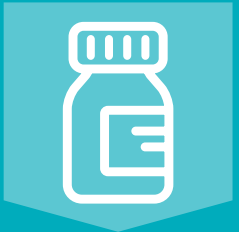

RIZMOIC ${ }^{\circledR}$ può essere usato CON O SENZA LASSATIVI. ${ }^{1}$
\#Ma si raccomanda di assumerlo ogni giorno alla stessa ora. OIC: Opioid-Induced Constipation, stipsi indotta da oppioidi.

\section{BIBLIOGRAFIA}

1. RCP RIZMOIC ${ }^{\oplus}$, versione corrente.

2. Modifica della Nota AIFA 90, Determina n. 459/2020, Gazzetta Ufficiale n 111 del 30 aprile 2020.
NOTA 90

La prescrizione a carico del SSN è limitata alle seguenti condizioni: ${ }^{2}$

- Soggetti in terapia cronica con oppiacei e diagnosi di costipazione indotta da oppiacei secondo i criteri di ROMA-IV che rispondano contemporaneamente alle seguenti caratteristiche:

- Terapia cronica e continuativa con oppiacei

- Resistenza al trattamento con almeno due lassativi di cui uno ad azione osmotica (la resistenza è definita come la mancata risposta dopo 3 giorni) ${ }^{2}$ 